\title{
SEMIGROUPS OF LOCAL HOMEOMORPHISMS AND INTERACTION GROUPS
}

\author{
R. EXEL* AND J. RENAULT**
}

\begin{abstract}
Given a semigroup of surjective local homeomorphisms on a compact space $X$ we consider the corresponding semigroup of *-endomorphisms on $C(X)$ and discuss the possibility of extending it to an interaction group, a concept recently introduced by the first named author. We may also define a transformation groupoid whose $\mathrm{C}^{*}$ algebra turns out to be isomorphic to the crossed product algebra for the interaction group. Several examples are considered, including one which gives rise to a slightly different construction and should be interpreted as being the $\mathrm{C}^{*}$-algebra of a certain polymorphism.
\end{abstract}

\section{Introduction.}

Recall from [6] that an interaction group consists of a triple $(A, G, V)$, where $A$ is a unital $\mathrm{C}^{*}$-algebra, $G$ is a discrete group, and $V=\left\{V_{g}\right\}_{g \in G}$ is a collection of positive, unit preserving linear operators on $A$ satisfying

(i) $V_{1}=i d_{A}$

(ii) $V_{g} V_{h} V_{h^{-1}}=V_{g h} V_{h^{-1}}$,

(iii) $V_{g^{-1}} V_{g} V_{h}=V_{g^{-1}} V_{g h}$,

(iv) $V_{g}(a b)=V_{g}(a) V_{g}(b)$, for every $a \in A$, and every $b$ in the range of $V_{g^{-1}}$.

This concept is a generalization of automorphism groups designed to deal with dynamical systems in which the transformations involved are not invertible and perhaps not even single valued.

We give examples of interaction groups arising from actions of semigroups and identify, in these examples, the crossed product algebra [6:6.2] as a groupoid $\mathrm{C}^{*}$-algebra.

Most of our examples are constructed as follows: let $X$ be a compact topological space, let $G$ be a discrete group, and let $\theta$ be a right action of a given subsemigroup $P \subseteq G$ on $X$ by means of surjective local homeomorphisms. The usual process of dualization, namely:

$$
\alpha_{n}(f)=f \circ \theta_{n}, \quad \forall n \in P, \quad \forall f \in C(X),
$$

defines an action $\alpha$ of $P$ on $C(X)$ by endomorphisms. The first hurdle that we face is to find an interaction group $V$ of $G$ on $A$ such that $V_{n}=\alpha_{n}$, for all $n \in P$.

2000 Mathematics Subject Classification: 46L55.

* Partially supported by CNPq and CAPES/COFECUB.

** Partially supported by CAPES/COFECUB.

Date: 23 Aug 2006. 
If such an interaction group is to be found then $V_{n^{-1}}$ must necessarily be a transfer operator [5:2.1] for $\alpha_{n}$, and hence we begin our search by looking for operators $L_{n}$ to fulfil this role. They must necessarily have the form

$$
\left.L_{n}(f)\right|_{y}=\sum_{\theta_{n}(x)=y} \omega(n, x) f(x), \quad \forall f \in C(X), \quad \forall y \in X,
$$

where $\omega: P \times X \rightarrow \mathbb{R}_{+}$is continuous in the second variable, and normalized in the sense that $\sum_{\theta_{n}(x)=y} \omega(n, x)=1$, for every $n \in P$ and $y \in X$.

Another necessary condition for the existence of the interaction group $V$ is that $L_{n}$ be anti-multiplicative in the semigroup variable " $n$ ", which amounts to $\omega$ satisfying

$$
\omega(n m, x)=\omega(n, x) \omega\left(m, \theta_{n}(x)\right), \quad \forall m, n \in P, \quad \forall x \in X .
$$

In other words $\omega$ must be a cocycle for the semigroup action. Assuming that $G=P^{-1} P$, and that $\omega$ satisfies still another coherence condition, we eventually prove that the formula

$$
\left.V_{g}(f)\right|_{y}=\sum_{\theta_{n}(x)=y} \omega(n, x) f\left(\theta_{m}(x)\right), \quad g=n^{-1} m, \quad \forall f \in C(X), \quad \forall y \in X
$$

does indeed give an interaction group extending $\alpha$.

Notice that the above formula amounts to a weighted average of $f$ on $\theta_{m}\left(\theta_{n}^{-1}(\{y\})\right)$, and hence it is a probabilistic version of the multi-valued map $f \circ \theta_{m} \circ \theta_{n}^{-1}$, which could also be written as $f \circ \theta_{n^{-1} m}$, since $\theta$ is a right action, except that $\theta_{n^{-1} m}$ has no meaning under the present hypothesis.

Motivated by $[\mathbf{1 0}],[\mathbf{2}]$ and $[\mathbf{3}]$ we define the transformation groupoid relative to the dynamical system $(X, P, \theta)$ to be

$$
\mathcal{G}=\left\{(x, g, y) \in X \times G \times X: \exists n, m \in P, g=n m^{-1}, \theta_{n}(x)=\theta_{m}(y)\right\},
$$

under the operations

$$
(x, g, y)(y, h, z)=(x, g h, z), \quad \text { and } \quad(x, g, y)^{-1}=\left(y, g^{-1}, x\right) .
$$

When all of the favorable conditions are present, in which case the interaction group is available, we show that the crossed product $C(X) \rtimes_{V} G$ and the groupoid $\mathrm{C}^{*}$-algebra $C^{*}(\mathcal{G})$ are naturally isomorphic.

We then present a series of examples to which our results may be applied. Closing our list of examples we look at the case of certain polymorphisms, a situation that does not precisely fit within our general framework, but for which we may also consider both the interaction group and the transformation groupoid, and prove the isomorphism between the crossed product and the groupoid $\mathrm{C}^{*}$-algebra.

It is interesting to notice that the existence of the interaction group extending a given semigroup action depends on the existence of cocycles satisfying suitable coherence 
conditions (see (2.7) below). On the other hand the transformation groupoid, and therefore also the associated groupoid $\mathrm{C}^{*}$-algebra may be defined irrespectively of the existence of cocycles. Since the coherence condition mentioned above does not seem to be studied in a systematic way, it might be an interesting project to determine, from the point of view of Dynamical Systems, whether or not a semigroup action admitting a coherent cocycle possesses special properties at all.

Based on the the concept of cellular automata we present, in our last section, an example of a semigroup action of $\mathbb{N} \times \mathbb{N}$ on Bernoulli's space which fails to admit a (nonzero) coherent cocycle. We believe that a further study of this example might give some insight on this so far fuzzy situation. Moreover it seems that this example is at odds with the last Proposition in $[\mathbf{2}]$.

\section{Semigroup actions.}

Given a compact space $X$ we will let $\operatorname{End}(X)$ denote the semigroup of all surjective local homeomorphisms

$$
T: X \rightarrow X
$$

under the composition law.

Let $G$ be a group and let $P$ be a subsemigroup (always assumed to contain 1 ) of $G$. By a right action of $P$ on $X$ we shall mean a map

$$
\theta: P \rightarrow \operatorname{End}(X)
$$

such that $\theta(1)=i d_{X}$, and $\theta_{n} \theta_{m}=\theta_{m n}$, for all $n$ and $m$ in $P$ (please notice the order reversal).

Given such a right action $\theta$, and denoting by $\operatorname{End}(C(X))$ the semigroup of injective unital *-endomorphisms of the algebra $C(X)$, we will let

$$
\alpha: P \rightarrow \operatorname{End}(C(X))
$$

be given by

$$
\alpha_{n}(f)=f \circ \theta_{n}, \quad \forall n \in P, \quad \forall f \in C(X) .
$$

It is immediate to verify that $\alpha_{n} \alpha_{m}=\alpha_{n m}$, for all $n$ and $m$ in $P$, so that $\alpha$ becomes a semigroup action of $P$ on $C(X)$.

We wish to introduce a transfer operator (see [5:2.1]) $L_{n}$ for each $\alpha_{n}$ so we fix a map

$$
\omega: P \times X \rightarrow \mathbb{R}_{+},
$$

which is continuous in the second variable, and such that

$$
\sum_{\theta_{n}(x)=y} \omega(n, x)=1, \quad \forall n \in P, \quad \forall y \in X .
$$


Given $n \in P$ and $f \in C(X)$, let

$$
\left.L_{n}(f)\right|_{y}=\sum_{\theta_{n}(x)=y} \omega(n, x) f(x), \quad \forall y \in X
$$

It is easy to show that $L_{n}(f) \in C(X)$ and that $L_{n}$ defines a transfer operator for $\alpha_{n}$, in the sense that $L_{n}$ is a positive linear operator on $C(X)$ satisfying $L_{n}(1)=1$, and

$$
L_{n}\left(f \alpha_{n}(g)\right)=L_{n}(f) g, \quad \forall f, g \in C(X) .
$$

2.2. Proposition. Suppose that

$$
\omega(n m, x)=\omega(n, x) \omega\left(m, \theta_{n}(x)\right), \quad \forall m, n \in P, \quad \forall x \in X
$$

Then $L_{n m}=L_{m} L_{n}$.

Proof. Given $f \in C(X)$ we have

$$
\begin{gathered}
\left.L_{m} L_{n}(f)\right|_{z}=\sum_{\theta_{m}(y)=z} \omega(m, y) \sum_{\theta_{n}(x)=y} \omega(n, x) f(x)= \\
=\sum_{\theta_{m}(y)=z} \sum_{\theta_{n}(x)=y} \omega\left(m, \theta_{n}(x)\right) \omega(n, x) f(x)=\sum_{\theta_{n m}(x)=z} \omega(n m, x) f(x)=\left.L_{n m}(f)\right|_{z} .
\end{gathered}
$$

2.3. Definition. A map $\omega: P \times X \rightarrow \mathbb{R}_{+}$which is continuous in the second variable and which satisfies both (2.1) and the condition in (2.2) will be called a normalized cocycle.

From now on we will assume that $\omega$ is a normalized cocycle.

Given $n \in P$ and $y \in X$ let us denote by $C_{y}^{n}$ the set

$$
C_{y}^{n}=\left\{x \in X: \theta_{n}(x)=\theta_{n}(y)\right\}
$$

One may then show that the range of $\alpha_{n}$ consists of the functions $f \in C(X)$ which are constant on $C_{y}^{n}$ for every $y$. Let

$$
E_{n}=\alpha_{n} \circ L_{n}
$$

or, in more explicit terms,

$$
\left.E_{n}(f)\right|_{y}=\sum_{x \in C_{y}^{n}} \omega(n, x) f(x), \quad \forall f \in C(X), \quad \forall y \in X
$$

It is an easy exercise to show that $E_{n}$ is a conditional expectation from $C(X)$ onto the range of $\alpha_{n}$. 
2.4. Lemma. Let $m, n \in P$. Then the following are equivalent

(i) $E_{n}$ and $E_{m}$ commute.

(ii) For every $x, z \in X$ we have that

$$
\sum_{y \in C_{x}^{m} \cap C_{z}^{n}} \omega(n, y) \omega(m, x)=\sum_{y \in C_{x}^{n} \cap C_{z}^{m}} \omega(m, y) \omega(n, x) .
$$

Proof. In this proof we will write

$$
\left.E_{n}(f)\right|_{y}=\sum_{x \in X}\left[\theta_{n}(x)=\theta_{n}(y)\right] \omega(n, x) f(x)
$$

using brackets to mean boolean value and observing that, although the sum is indexed on the infinite set $X$, only finitely many summands are non-zero. We then have for every $z$ in $X$ that

$$
\begin{gathered}
\left.E_{n} E_{m}(f)\right|_{z}=\sum_{y \in X} \sum_{x \in X}\left[\theta_{n}(y)=\theta_{n}(z)\right]\left[\theta_{m}(x)=\theta_{m}(y)\right] \omega(n, y) \omega(m, x) f(x)= \\
=\sum_{x \in X}\left(\sum_{y \in X}\left[\theta_{n}(y)=\theta_{n}(z)\right]\left[\theta_{m}(x)=\theta_{m}(y)\right] \omega(n, y) \omega(m, x)\right) f(x)= \\
=\sum_{x \in X}\left(\sum_{y \in C_{x}^{m} \cap C_{z}^{n}} \omega(n, y) \omega(m, x)\right) f(x) .
\end{gathered}
$$

Interchanging $n$ and $m$ we see that

$$
\left.E_{m} E_{n}(f)\right|_{z}=\sum_{x \in X}\left(\sum_{y \in C_{x}^{n} \cap C_{z}^{m}} \omega(m, y) \omega(n, x)\right) f(x),
$$

from where the conclusion follows easily.

We would like to further elaborate on condition (2.4.ii). Given a finite subset $S \subseteq X$, and $n \in P$, let us denote by

$$
W_{n}(S)=\sum_{y \in S} \omega(n, y)
$$

Also, given $x, z \in X$, and $n, m \in P$, let

$$
C_{x, z}^{n, m}=C_{x}^{n} \cap C_{z}^{m} .
$$

Condition (2.4.ii) may then be rephrased as follows: 
2.7. Definition. We shall say that a cocycle $\omega$ is coherent if for all $x, z \in X$, and for all $m, n \in P$, one has that

$$
\omega(m, x) W_{n}\left(C_{x, z}^{m, n}\right)=\omega(n, x) W_{m}\left(C_{x, z}^{n, m}\right)
$$

We thus arrive at the first point of contact with the theory of interaction groups.

\subsection{Theorem. Let}

(i) $G$ be a group and $X$ be a compact space,

(ii) $P$ be a subsemigroup of $G$ such that $G=P^{-1} P$,

(iii) $\theta: P \rightarrow \operatorname{End}(X)$ be a right action, and

(iv) $\omega$ be a normalized coherent cocycle.

Then there exists a unique interaction group $V=\left\{V_{g}\right\}_{g \in G}$ on $C(X)$, such that $V_{n}=\alpha_{n}$, and $V_{n^{-1}}=L_{n}$, for all $n$ in $P$. Moreover, if $g=n^{-1} m$, with $n, m \in P$, then $V_{g}=L_{n} \alpha_{m}$, or more explicitly

$$
\left.V_{g}(f)\right|_{y}=\sum_{\theta_{n}(x)=y} \omega(n, x) f\left(\theta_{m}(x)\right), \quad \forall f \in C(X), \quad \forall y \in X
$$

Proof. Follows immediately from the results above and [6:13.3]. The formula for $V_{g}$ above follows from the fact that $L_{n} \alpha_{n}=i d_{C(X)}$, and hence

$$
V_{g}=L_{n} \alpha_{n} V_{n^{-1} m}=V_{n^{-1}} V_{n} V_{n^{-1} m}=V_{n^{-1}} V_{m}=L_{n} \alpha_{m}
$$

\section{The transformation groupoid.}

Given an interaction group, such as one might obtain from Theorem (2.8), we may consider its crossed product algebra

$$
C(X) \rtimes_{V} G,
$$

as introduced by $[6: 6.2]$. We would like to show that this algebra coincides with the $\mathrm{C}^{*}$-algebra of a transformation groupoid naturally constructed from the given dynamical system, which we introduce in this section. This is essentially the groupoid studied in [10], $[\mathbf{2}],[\mathbf{1}]$ but, as we shall see, the extension to actions of arbitrary semigroups requires some further work.

For the time being we will assume that $G$ is a group, $P$ is a subsemigroup of $G$, and

$$
\theta: P \rightarrow \operatorname{End}(X)
$$

is a right action of $P$ on the compact space $X$. 
3.1. Proposition. Suppose that $P^{-1} P \subseteq P P^{-1}$. Then the set

$$
\mathcal{G}=\left\{(x, g, y) \in X \times G \times X: \exists n, m \in P, g=n m^{-1}, \theta_{n}(x)=\theta_{m}(y)\right\}
$$

is a groupoid under the operations

$$
(x, g, y)(y, h, z)=(x, g h, z), \quad \text { and } \quad(x, g, y)^{-1}=\left(y, g^{-1}, x\right) .
$$

Proof. Let us prove that the operations defined above do indeed give elements of $\mathcal{G}$. With respect to the inversion suppose that $(x, g, y) \in \mathcal{G}$ and let $n, m \in P$ be such that $g=n m^{-1}$ and $\theta_{n}(x)=\theta_{m}(y)$. Then obviously $g^{-1}=m n^{-1}$ and $\theta_{m}(y)=\theta_{n}(x)$, so $\left(y, g^{-1}, x\right) \in \mathcal{G}$.

If moreover $(y, h, z) \in \mathcal{G}$, let $p, q \in P$ be such that $h=p q^{-1}$ and $\theta_{p}(y)=\theta_{q}(z)$. Given that $P^{-1} P \subseteq P P^{-1}$, write $m^{-1} p=u v^{-1}$, with $u, v \in P$. So

$$
\theta_{n u}(x)=\theta_{u}\left(\theta_{n}(x)\right)=\theta_{u}\left(\theta_{m}(y)\right)=\theta_{m u}(y)=\theta_{p v}(y)=\theta_{v}\left(\theta_{p}(y)\right)=\theta_{v}\left(\theta_{q}(z)\right)=\theta_{q v}(z) .
$$

In addition

$$
n u(q v)^{-1}=n u v^{-1} q^{-1}=n m^{-1} p q^{-1}=g h,
$$

thus showing that $(x, g h, z) \in \mathcal{G}$. We leave it for the reader to check the other groupoid axioms.

The next result deals with the topological aspects of $\mathcal{G}$. We thank for this Trent Yeend, who proved a similar result in $[\mathbf{1 1}: 3.6]$.

3.2. Proposition. Suppose that $P^{-1} P \subseteq P P^{-1}$. For every $n, m \in P$, and for every open sets $A, B \subseteq X$, let

$$
\Sigma(n, m, A, B)=\left\{(x, g, y) \in \mathcal{G}: g=n m^{-1}, \theta_{n}(x)=\theta_{m}(y), x \in A, y \in B\right\} .
$$

Then the collection of all such subsets is a basis for a topology on $\mathcal{G}$, with respect to which it is a locally compact étale groupoid.

Proof. It is obvious that the $\Sigma(n, m, A, B)$ cover $\mathcal{G}$. Next we must verify that if

$$
(x, g, y) \in \Sigma\left(n_{1}, m_{1}, A_{1}, B_{1}\right) \cap \Sigma\left(n_{2}, m_{2}, A_{2}, B_{2}\right)
$$

then there exists some $\Sigma(n, m, A, B)$ such that

$$
(x, g, y) \in \Sigma(n, m, A, B) \subseteq \Sigma\left(n_{1}, m_{1}, A_{1}, B_{1}\right) \cap \Sigma\left(n_{2}, m_{2}, A_{2}, B_{2}\right) .
$$

By hypothesis there exists $p_{1}, p_{2} \in P$ such that $n_{2}^{-1} n_{1}=p_{2} p_{1}^{-1}$.

For $i=1,2$, let $U_{i}$ be an open set with

$$
\theta_{n_{i}}(x)=\theta_{m_{i}}(y) \in U_{i}
$$


and such that $\theta_{p_{i}}$ is injective on $U_{i}$. Defining

- $n=n_{1} p_{1}, \quad m=m_{1} p_{1}$,

- $A=A_{1} \cap A_{2} \cap \theta_{n_{1}}^{-1}\left(U_{1}\right) \cap \theta_{n_{2}}^{-1}\left(U_{2}\right)$,

- $B=B_{1} \cap B_{2} \cap \theta_{m_{1}}^{-1}\left(U_{1}\right) \cap \theta_{m_{2}}^{-1}\left(U_{2}\right)$,

we claim that $\Sigma(n, m, A, B)$ has the desired properties. To see this first observe that $g=n_{1} m_{1}^{-1}=n_{2} m_{2}^{-1}$, whence

$$
m=m_{1} p_{1}=m_{2} n_{2}^{-1} n_{1} p_{1}=m_{2} p_{2} p_{1}^{-1} p_{1}=m_{2} p_{2},
$$

and likewise

$$
n=n_{1} p_{1}=n_{2} p_{2} \text {. }
$$

Thus if $\left(x^{\prime}, g, y^{\prime}\right) \in \Sigma(n, m, A, B)$ we have for $i=1,2$ that

$$
\theta_{p_{i}}\left(\theta_{n_{i}}\left(x^{\prime}\right)\right)=\theta_{n_{i} p_{i}}\left(x^{\prime}\right)=\theta_{n}\left(x^{\prime}\right)=\theta_{m}\left(y^{\prime}\right)=\theta_{m_{i} p_{i}}\left(y^{\prime}\right)=\theta_{p_{i}}\left(\theta_{m_{i}}\left(y^{\prime}\right)\right) .
$$

Moreover notice that

$$
\begin{aligned}
x^{\prime} \in A \subseteq \theta_{n_{i}}^{-1}\left(U_{i}\right) & \Longrightarrow \theta_{n_{i}}\left(x^{\prime}\right) \in U_{i}, \\
y^{\prime} \in B \subseteq \theta_{m_{i}}^{-1}\left(U_{i}\right) & \Longrightarrow \theta_{m_{i}}\left(y^{\prime}\right) \in U_{i} .
\end{aligned}
$$

Since $\theta_{p_{i}}$ is injective on $U_{i}$ we have that $\theta_{n_{i}}\left(x^{\prime}\right)=\theta_{m_{i}}\left(y^{\prime}\right)$, which implies that $\left(x^{\prime}, g, y^{\prime}\right) \in$ $\Sigma\left(n_{i}, m_{i}, A_{i}, B_{i}\right)$, thus showing that

$$
\Sigma(n, m, A, B) \subseteq \Sigma\left(n_{1}, m_{1}, A_{1}, B_{1}\right) \cap \Sigma\left(n_{2}, m_{2}, A_{2}, B_{2}\right) .
$$

In order to see that $(x, g, y) \in \Sigma(n, m, A, B)$, notice that

$$
\theta_{n}(x)=\theta_{n_{1} p_{1}}(x)=\theta_{p_{1}}\left(\theta_{n_{1}}(x)\right)=\theta_{p_{1}}\left(\theta_{m_{1}}(y)\right)=\theta_{m_{1} p_{1}}(y)=\theta_{m}(y)
$$

It is also easy to see that $x \in A$ and $y \in B$, so indeed $(x, g, y) \in \Sigma(n, m, A, B)$.

Before proving that $\mathcal{G}$ is locally compact let for every $n, m \in P$

$$
E(n, m)=\left\{(x, y) \in X \times X: \theta_{n}(x)=\theta_{m}(y)\right\},
$$

considered as a topological subspace of $X \times X$. We claim that the map

$$
\iota:(x, y) \in E(n, m) \mapsto\left(x, n m^{-1}, y\right) \in \mathcal{G}
$$

is continuous. To prove that $\iota$ is continuous at a point $\left(x_{0}, y_{0}\right) \in E(n, m)$, let $W$ be a neighborhood of $\left(x_{0}, n m^{-1}, y_{0}\right)$ in $\mathcal{G}$. Then there exists a basic open set, say $\Sigma(k, l, A, B)$, such that

$$
\left(x_{0}, n m^{-1}, y_{0}\right) \in \Sigma(k, l, A, B) \subseteq W .
$$


One then must have $n m^{-1}=k l^{-1}$. Choose $p, q \in P$ such that $k^{-1} n=p q^{-1}$, and let $W$ be an open neighborhood of $\theta_{k}\left(x_{0}\right)=\theta_{l}\left(y_{0}\right)$, such that $\theta_{p}$ is injective on $W$. Letting

- $U=A \cap \theta_{k}^{-1}(W)$,

- $V=B \cap \theta_{l}^{-1}(W)$, and

- $Z=(U \times V) \cap E(n, m)$,

observe that $Z$ is an open subset of $E(n, m)$ containing $\left(x_{0}, y_{0}\right)$. We claim that $\iota(Z) \subseteq W$. In fact, if $(x, y) \in Z$ then

$$
\theta_{p}\left(\theta_{k}(x)\right)=\theta_{k p}(x)=\theta_{n q}(x)=\theta_{q}\left(\theta_{n}(x)\right)=\theta_{q}\left(\theta_{m}(y)\right)=\theta_{m q}(y)=\ldots
$$

Observe that $m q=l k^{-1} n q=l p q^{-1} q=l p$, so the above equals

$$
\ldots=\theta_{l p}(y)=\theta_{p}\left(\theta_{l}(y)\right)
$$

Summarizing we have that $\theta_{p}\left(\theta_{k}(x)\right)=\theta_{p}\left(\theta_{l}(y)\right)$, but since

$$
\begin{aligned}
& x \in U \subseteq \theta_{k}^{-1}(W) \Longrightarrow \theta_{k}(x) \in W, \\
& y \in V \subseteq \theta_{l}^{-1}(W) \Longrightarrow \theta_{l}(y) \in W,
\end{aligned}
$$

and since $\theta_{p}$ is injective on $W$, we have that $\theta_{k}(x)=\theta_{l}(y)$, from where one concludes that

$$
\iota(x, y)=\left(x, n m^{-1}, y\right)=\left(x, k l^{-1}, y\right) \in \Sigma(k, l, A, B) \subseteq W
$$

thus proving that $\iota(Z) \subseteq W$, and hence that $\iota$ is continuous.

Viewing $\iota$ as a map

$$
\iota: E(n, m) \rightarrow \Sigma(n, m, X, X)=: \Sigma(n, m)
$$

we thus see that $\iota$ is a homeomorphism onto $\Sigma(n, m)$, because $E(n, m)$ is compact and $\Sigma(n, m)$ is Hausdorff. Since $\Sigma(n, m)$ is open by definition we deduce that $\mathcal{G}$ is locally compact. We leave the verification of the remaining properties to the reader. 


\section{A semigroup of isometries.}

Our major goal is to prove the isomorphism between $C(X) \rtimes_{V} G$ and $C^{*}(\mathcal{G})$. In order for both of these algebras to be defined we may invoke (2.8) and (3.2) and hence we must restrict ourselves to a situation in which all of the relevant hypothesis are satisfied. We therefore suppose throughout that we are under the following:

\subsection{Standing Hypotheses.}

(i) $G$ is a discrete group and $P$ is a subsemigroup such that $1 \in P$, and $G=P^{-1} P=$ $P P^{-1}$,

(ii) $\theta: P \rightarrow \operatorname{End}(X)$ is right action of $P$ on the compact space $X$, and

(iii) $\omega$ is a normalized coherent cocycle which never vanishes.

The proof of the aforementioned isomorphism will be based on the construction of a suitable covariant representation $(\pi, \sigma)$ [6:4.1] of our interaction group. In this section we construct a semigroup of isometries which will later be used to construct the partial representation $\sigma$.

4.2. Proposition. For each $n \in P$, let $S_{n}$ be the element of $C^{*}(\mathcal{G})$ given by

$$
S_{n}(x, g, y)=\omega(n, x)^{1 / 2}[g=n]\left[\theta_{n}(x)=y\right],
$$

where the brackets correspond to boolean value. Then $\left\{S_{n}\right\}_{n \in P}$ is a semigroup of isometries in $C^{*}(\mathcal{G})$.

Proof. In order to verify that $S_{n}$ is an isometry for every $n$ first observe that

$$
\begin{aligned}
S_{n}^{*}(x, g, y) & =\overline{S_{n}\left(y, g^{-1}, x\right)}= \\
& =\omega(n, y)^{1 / 2}\left[g=n^{-1}\right]\left[x=\theta_{n}(y)\right] .
\end{aligned}
$$

Therefore

$$
\begin{gathered}
\left.S_{n}^{*} S_{n}\right|_{(x, g, y)}=\sum_{(x, h, z) \in \mathcal{G}} S_{n}^{*}(x, h, z) S_{n}\left(z, h^{-1} g, y\right)= \\
=\sum_{(x, h, z) \in \mathcal{G}} \omega(n, z)^{1 / 2}\left[h=n^{-1}\right]\left[x=\theta_{n}(z)\right] \omega(n, z)^{1 / 2}\left[h^{-1} g=n\right]\left[\theta_{n}(z)=y\right]= \\
=\sum_{\theta_{n}(z)=x} \omega(n, z)[g=1]\left[\theta_{n}(z)=y\right]= \\
=[g=1][x=y] \sum_{\theta_{n}(z)=x} \omega(n, z)=[g=1][x=y],
\end{gathered}
$$

thus showing that $S_{n}^{*} S_{n}=1$. We next show that $S_{n} S_{m}=S_{n m}$, for every $n, m \in P$.

$$
\left.S_{n} S_{m}\right|_{(x, g, y)}=\sum_{(x, h, z) \in \mathcal{G}} S_{n}(x, h, z) S_{m}\left(z, h^{-1} g, y\right)=
$$




$$
\begin{gathered}
=\sum_{(x, h, z) \in \mathcal{G}} \omega(n, x)^{1 / 2}[h=n]\left[\theta_{n}(x)=z\right] \omega(m, z)^{1 / 2}\left[h^{-1} g=m\right]\left[\theta_{m}(z)=y\right]= \\
=\omega(n, x)^{1 / 2} \omega\left(m, \theta_{n}(x)\right)^{1 / 2}\left[n^{-1} g=m\right]\left[\theta_{m}\left(\theta_{n}(x)\right)=y\right]= \\
=\omega(n m, x)^{1 / 2}[g=n m]\left[\theta_{n m}(x)=y\right]=S_{n m}(x, g, y) .
\end{gathered}
$$

Observe that we have not used the fact that $\omega$ is coherent in the above proof.

Recall that, whenever $\mathcal{G}$ is an étale groupoid, with unit space $\mathcal{G}^{(0)}$, the algebra $C_{0}\left(\mathcal{G}^{(0)}\right)$ sits naturally as a subalgebra of $C^{*}(\mathcal{G})$. In our case $\mathcal{G}^{(0)}=X$, so we will henceforth identify $C(X)$ with the corresponding subalgebra of $C^{*}(\mathcal{G})$.

We now wish to show that $C^{*}(\mathcal{G})$ is generated, as a $\mathrm{C}^{*}$-algebra, by

$$
C(X) \cup\left\{S_{n}: n \in P\right\}
$$

In preparation for this we will occasionally consider the pointwise (as opposed to the convolution) product for functions on $\mathcal{G}$. In order to avoid confusion we will denote pointwise product by $f \cdot g$, keeping the usual juxtaposition notation for the convolution product.

In what follows we will say that a subset $E \subseteq \mathcal{G}$ is an $r$-section when $r$ (the groupoid range map) is injective on $E$.

4.3. Lemma. Let $f$ and $g$ be continuous complex valued functions on $\mathcal{G}$, and suppose that $\operatorname{supp}(f)$ is a compact $r$-section. Then there exists $u \in C(X)$ such that

$$
g \cdot f=u f
$$

Proof. Let $K=\operatorname{supp}(f)$, and let $r^{-1}$ refer to the inverse of the restriction

$$
r: K \rightarrow r(K)
$$

Since $r$ is continuous and $K$ is compact we have that $r^{-1}$ is continuous. Define $u_{0}$ on $r(K)$ by $u_{0}=g \circ r^{-1}$ and use Tietze's Theorem to extend $u_{0}$ to a continuous function $u$ on $X$. Then, for every $\gamma \in \mathcal{G}$ we have

$$
(u f)(\gamma)=u(r(\gamma)) f(\gamma)=g(\gamma) f(\gamma)=(g \cdot f)(\gamma)
$$

The following elementary result will be useful later and is included for completeness.

4.4. Lemma. Let $\Omega$ be a locally compact space and let $\mathcal{F} \subseteq C_{c}(\Omega)$ be such that for every $x \in \Omega$ there is an $f \in \mathcal{F}$ such that $f(x) \neq 0$. Then

$$
C_{c}(\Omega)=\operatorname{span}\left\{g f: g \in C_{c}(\Omega), f \in \mathcal{F}\right\}
$$


Proof. Given $h \in C_{c}(\Omega)$ let $K=\operatorname{supp}(h)$. For each $x \in K$ choose $f_{x} \in \mathcal{F}$ such that $f_{x}(x) \neq 0$. Setting $U_{x}=\left\{y \in \Omega: f_{x}(y) \neq 0\right\}$ we have that $\left\{U_{x}\right\}_{x \in K}$ is an open cover of $K$, so

$$
K \subseteq U_{x_{1}} \cup \ldots U_{x_{n}}
$$

for suitable $x_{1}, \ldots, x_{n} \in K$. For simplicity of notation set $f_{i}:=f_{x_{i}}$ and observe that $\sum_{i=1}^{n}\left|f_{i}\right|^{2}>0$ on $K$. By Tietze's Theorem choose $g \in C(\Omega)$ such that

$$
g(x)=\left(\sum_{i=1}^{n}\left|f_{i}(x)\right|^{2}\right)^{-1}, \quad \forall x \in K .
$$

It follows that $\sum_{i=1}^{n}\left(h g \bar{f}_{i}\right) f_{i}=h$.

We thus arrive at a main result:

4.5. Proposition. $C_{c}(\mathcal{G})=\operatorname{span}\left\{u S_{n} S_{m}^{*} v: n, m \in P, u, v \in C(X)\right\}$.

Proof. Let $\mathcal{F} \subseteq C_{c}(\mathcal{G})$ be the collection of all functions of the form $S_{n} S_{m}^{*} v$, where $n, m \in P$, and $v \in C(X)$ is such that $\theta_{m}$ is injective on $\operatorname{supp}(v)$.

Observe that the support of $S_{n} S_{m}^{*} v$, is contained in $\Sigma(n, m, X, \operatorname{supp}(v))$. In fact, if

$$
0 \neq\left. S_{n} S_{m}^{*} v\right|_{(x, g, y)}=v(y) \omega(n, x)^{1 / 2} \omega(m, y)^{1 / 2}\left[g=n m^{-1}\right]\left[\theta_{n}(x)=\theta_{m}(y)\right]
$$

then $y \in \operatorname{supp}(v), g=n m^{-1}$, and $\theta_{n}(x)=\theta_{m}(y)$, which says that $(x, g, y)$ belongs to the indicated set.

Notice that $\Sigma(n, m, A, B)$ is an $r$-section whenever $\theta_{m}$ is injective on $B$. In fact, if

$$
\left(x, n m^{-1}, y\right),\left(x^{\prime}, n m^{-1}, y^{\prime}\right) \in \Sigma(n, m, A, B),
$$

and $x=x^{\prime}$, then

$$
\theta_{m}(y)=\theta_{n}(x)=\theta_{n}\left(x^{\prime}\right)=\theta_{m}\left(y^{\prime}\right),
$$

so that $y=y^{\prime}$ because $\theta_{m}$ is injective on $B$. It follows that the support of every $f \in \mathcal{F}$ is an $r$-section.

Given that $\mathcal{F}$ satisfies the hypothesis of (4.4), as one may easily verify, we conclude that any $h \in C_{c}(\mathcal{G})$ may be written as

$$
h=\sum_{i=1}^{n} g_{i} \cdot f_{i},
$$

where $g_{i} \in C_{c}(\mathcal{G})$, and $f_{i} \in \mathcal{F}$. But since $f_{i}$ is supported on an $r$-section, as seen above, we have that $g_{i} \cdot f_{i}=u_{i} f_{i}$, for some $u_{i} \in C(X)$, by (4.3). Writing $f_{i}=S_{n_{i}} S_{m_{i}}^{*} v_{i}$, we have that

$$
h=\sum_{i=1}^{n} u_{i} S_{n_{i}} S_{m_{i}}^{*} v_{i}
$$

as required. 


\section{A partial representation.}

We next wish to study the possibility of extending $S$ to a partial representation $\sigma$ of $G$ in $C^{*}(\mathcal{G})$ which will be part of the covariant representation we are looking for. The following abstract result will suit us well:

5.1. Proposition. Let $P$ be a subsemigroup of a group $G$ such that $G=P^{-1} P$, and let $S: P \rightarrow B$ be a semigroup of isometries in a $C^{*}$-algebra $B$. If $S_{n} S_{n}^{*}$ and $S_{m} S_{m}^{*}$ commute for all $n$ and $m$ in $P$ then there exists a unique ${ }^{*}$-partial representation

$$
\sigma: G \rightarrow B
$$

such that $\sigma_{n}=S_{n}$, for every $n \in P$. Moreover, if $g=n^{-1} m$, with $n, m \in P$, then $\sigma_{g}=S_{n}^{*} S_{m}$.

Proof. Supposing that $\sigma$ exists let us first address the very last part of the statement and hence also uniqueness. Given $g=n^{-1} m$, observe that $\sigma_{m^{-1}} \sigma_{m}=S_{m}^{*} S_{m}=1$, so

$$
\sigma_{g}=\sigma_{n^{-1} m} \sigma_{m^{-1}} \sigma_{m}=\sigma_{n^{-1}} \sigma_{m}=S_{n}^{*} S_{m}
$$

With respect to existence, for every $g \in G$, write $g=n^{-1} m$, with $n, m \in P$, and set

$$
\sigma_{g}=S_{n}^{*} S_{m}
$$

Let us prove that this is well defined: if $g$ can also be written as $g=p^{-1} q$, we claim that there exists $x, y, u, v \in P$ such that

$$
\left\{\begin{array}{c}
x p=u, \\
x q=v, \\
y n=u, \\
y m=v .
\end{array}\right.
$$

In fact, use the hypothesis to write $n p^{-1}=y^{-1} x$, with $x, y \in P$, and set $u=x p$, and $v=x q$. Then the first two equations hold true and one clearly has that $y n=x p=u$, while

$$
y m=y n n^{-1} m=x p g=x p p^{-1} q=x q=v .
$$

One therefore has that

$$
S_{n}^{*} S_{m}=S_{n}^{*} S_{y}^{*} S_{y} S_{m}=S_{y n}^{*} S_{y m}=S_{u}^{*} S_{v}=S_{x p}^{*} S_{x q}=S_{p}^{*} S_{x}^{*} S_{x} S_{q}=S_{p}^{*} S_{q}
$$

This proves that $\sigma$ is well defined. Let us now show the partial group law, that is,

$$
\sigma_{g} \sigma_{h} \sigma_{h^{-1}}=\sigma_{g h} \sigma_{h^{-1}}
$$


for all $g, h \in G$. Write $g=n^{-1} m$, and $h=p^{-1} q$, with $n, m, p, q \in P$. Pick $u, v \in P$ such that $p m^{-1}=v^{-1} u$ and notice that

$$
u m=v p .
$$

Replacing $(n, m)$ by $(u n, u m)$, and $(p, q)$ by $(v p, v q)$, we may then assume that $m=p$. We then have

$$
\sigma_{g} \sigma_{h} \sigma_{h^{-1}}=S_{n}^{*} S_{m} S_{m}^{*} S_{q} S_{q}^{*} S_{m}=S_{n}^{*} S_{q} S_{q}^{*} S_{m} S_{m}^{*} S_{m}=S_{n}^{*} S_{q} S_{q}^{*} S_{m}=\sigma_{g h} \sigma_{h^{-1}}
$$

We now wish to verify that the hypothesis of the above abstract result does indeed apply in our situation. Our next Lemma is designed to isolate the more technical aspects of that verification. In it we will make extensive use of the notation introduced in (2.5) and (2.6).

5.2. Lemma. Assuming (4.1) let $x, z \in X$. Then the expressions

(i) $W_{m}\left(C_{x, x}^{n, m}\right) W_{n}\left(C_{x, z}^{m, n}\right)$, and

(ii) $\sum_{y \in C_{x, z}^{m, n}} \omega(m, y)^{1 / 2} \omega(n, y)^{1 / 2} \omega(m, x)^{1 / 2} \omega(n, z)^{1 / 2}$

are invariant under exchanging the variables $n$ and $m$ in $P$.

Proof. With respect to (i) we have to prove that

$$
W_{m}\left(C_{x, x}^{n, m}\right) W_{n}\left(C_{x, z}^{m, n}\right)=W_{n}\left(C_{x, x}^{n, m}\right) W_{m}\left(C_{x, z}^{n, m}\right)
$$

For every $y$ we have by (2.7) that

$$
\omega(m, y) W_{n}\left(C_{y, z}^{m, n}\right)=\omega(n, y) W_{m}\left(C_{y, z}^{n, m}\right) .
$$

If $y \in C_{x, x}^{n, m}$ then $C_{y, z}^{m, n}=C_{x, z}^{m, n}$, and $C_{y, z}^{n, m}=C_{x, z}^{n, m}$, so the equation displayed above becomes

$$
\omega(m, y) W_{n}\left(C_{x, z}^{m, n}\right)=\omega(n, y) W_{m}\left(C_{x, z}^{n, m}\right) .
$$

Adding up both sides over all $y \in C_{x, x}^{n, m}$ we obtain (i).

Let us now deal with (ii). If $C_{x, z}^{m, n}=\emptyset$, then by $(2.7)$ we have that $C_{x, z}^{n, m}=\emptyset$ as well (because $\omega$ never vanishes) and hence the expression in (ii) vanishes regardless of the order in which we take $n$ and $m$. On the other hand, given $y \in C_{x, z}^{m, n}$, we have that $C_{x, z}^{n, m} \neq \emptyset$, again by $(2.7)$, so let $u \in C_{x, z}^{n, m}$.

Using (2.7) we have that

$$
\omega(m, y) W_{n}\left(C_{y, u}^{m, n}\right)=\omega(n, y) W_{m}\left(C_{y, u}^{n, m}\right)
$$

Notice that $C_{y, u}^{m, n}=C_{x, x}^{m, n}$, and $C_{y, u}^{n, m}=C_{z, z}^{n, m}$, so the above becomes

$$
\omega(m, y) W_{n}\left(C_{x, x}^{m, n}\right)=\omega(n, y) W_{m}\left(C_{z, z}^{n, m}\right) .
$$


Working with just the part of the expression in (ii) that involves the variable $y$, we have

$$
\begin{gathered}
\sum_{y \in C_{x, z}^{m, n}} \omega(m, y)^{1 / 2} \omega(n, y)^{1 / 2}= \\
=\sum_{y \in C_{x, z}^{m, n}} \omega(n, y)^{1 / 2} W_{m}\left(C_{z, z}^{n, m}\right)^{1 / 2} W_{n}\left(C_{x, x}^{m, n}\right)^{-1 / 2} \omega(n, y)^{1 / 2}= \\
=W_{m}\left(C_{z, z}^{n, m}\right)^{1 / 2} W_{n}\left(C_{x, x}^{m, n}\right)^{-1 / 2} \sum_{y \in C_{x, z}^{m, n}} \omega(n, y)= \\
=W_{m}\left(C_{z, z}^{n, m}\right)^{1 / 2} W_{n}\left(C_{x, x}^{m, n}\right)^{-1 / 2} W_{n}\left(C_{x, z}^{m, n}\right) .
\end{gathered}
$$

We thus conclude that (ii) equals

$$
\begin{gathered}
\omega(m, x)^{1 / 2} \omega(n, z)^{1 / 2} W_{m}\left(C_{z, z}^{n, m}\right)^{1 / 2} W_{n}\left(C_{x, x}^{m, n}\right)^{-1 / 2} \underline{W_{n}\left(C_{x, z}^{m, n}\right)}= \\
=\omega(m, x)^{1 / 2} \underline{W_{n}\left(C_{x, z}^{m, n}\right)^{1 / 2}} \omega(n, z)^{1 / 2} W_{m}\left(C_{z, z}^{n, m}\right)^{1 / 2} W_{n}\left(C_{x, x}^{m, n}\right)^{-1 / 2} \underline{W_{n}\left(C_{x, z}^{m, n}\right)^{1 / 2}} .
\end{gathered}
$$

Using (2.7), as it stands, we deduce that (5.2.2) equals

$$
\omega(n, x)^{1 / 2} W_{m}\left(C_{x, z}^{n, m}\right)^{1 / 2} \omega(n, z)^{1 / 2} W_{m}\left(C_{z, z}^{n, m}\right)^{1 / 2} W_{n}\left(C_{x, x}^{m, n}\right)^{-1 / 2} W_{n}\left(C_{x, z}^{m, n}\right)^{1 / 2}=\ldots
$$

Using (2.7) again, this time in the form

$$
\omega(n, z) W_{m}\left(C_{z, z}^{n, m}\right)=\omega(m, z) W_{n}\left(C_{z, z}^{n, m}\right)
$$

we conclude that (5.2.2) equals

$$
\omega(n, x)^{1 / 2} W_{m}\left(C_{x, z}^{n, m}\right)^{1 / 2} \omega(m, z)^{1 / 2} W_{n}\left(C_{z, z}^{n, m}\right)^{1 / 2} W_{n}\left(C_{x, x}^{m, n}\right)^{-1 / 2} W_{n}\left(C_{x, z}^{m, n}\right)^{1 / 2}
$$

We want to show this to equal (5.2.2) with $n$ and $m$ exchanged, namely

$$
\omega(n, x)^{1 / 2} \omega(m, z)^{1 / 2} W_{n}\left(C_{z, z}^{m, n}\right)^{1 / 2} W_{m}\left(C_{x, x}^{n, m}\right)^{-1 / 2} W_{m}\left(C_{x, z}^{n, m}\right)
$$

which turns out to be equivalent to verifying that

$$
W_{m}\left(C_{x, z}^{n, m}\right)^{1 / 2} W_{n}\left(C_{x, x}^{m, n}\right)^{-1 / 2} W_{n}\left(C_{x, z}^{m, n}\right)^{1 / 2}=W_{m}\left(C_{x, x}^{n, m}\right)^{-1 / 2} W_{m}\left(C_{x, z}^{n, m}\right),
$$

which happens to be just a rewriting of (i).

The more involving technical aspects taken care of, we may prove: 
5.3. Proposition. Under (4.1) there exists a unique partial representation

$$
\sigma: G \rightarrow C^{*}(\mathcal{G})
$$

such that $\sigma_{n}=S_{n}$, for every $n \in P$, where $\left\{S_{n}\right\}_{n \in P}$ is the semigroup of isometries in $C^{*}(\mathcal{G})$ given by (4.2). Moreover, if $g=n^{-1} m$, with $n, m \in P$, then $\sigma_{g}=S_{n}^{*} S_{m}$.

Proof. In view of (5.1) it is enough to verify that $S_{n} S_{n}^{*}$ and $S_{m} S_{m}^{*}$ commute for all $n$ and $m$ in $P$. Observe that, for all $(x, g, y) \in \mathcal{G}$, we have

$$
\begin{gathered}
S_{n} S_{n}^{*}(x, g, y)=\sum_{(x, h, z) \in \mathcal{G}} S_{n}(x, h, z) S_{n}^{*}\left(z, h^{-1} g, y\right)= \\
=\sum_{(x, h, z) \in \mathcal{G}} \omega(n, x)^{1 / 2}[h=n]\left[\theta_{n}(x)=z\right] \omega(n, y)^{1 / 2}\left[h^{-1} g=n^{-1}\right]\left[\theta_{n}(y)=z\right]= \\
=\omega(n, x)^{1 / 2} \omega(n, y)^{1 / 2}[g=1]\left[\theta_{n}(x)=\theta_{n}(y)\right] .
\end{gathered}
$$

Thus

$$
\begin{gathered}
S_{m} S_{m}^{*} S_{n} S_{n}^{*}(x, g, z)=\sum_{(x, h, y) \in \mathcal{G}} S_{m} S_{m}^{*}(x, h, y) S_{n} S_{n}^{*}\left(y, h^{-1} g, z\right)= \\
=\sum_{(x, h, y) \in \mathcal{G}} \omega(m, x)^{1 / 2} \omega(m, y)^{1 / 2}[h=1]\left[\theta_{m}(x)=\theta_{m}(y)\right] \\
\omega(n, y)^{1 / 2} \omega(n, z)^{1 / 2}\left[h^{-1} g=1\right]\left[\theta_{n}(y)=\theta_{n}(z)\right]= \\
=[g=1] \sum_{y \in C_{x, z}^{m, n}} \omega(m, x)^{1 / 2} \omega(m, y)^{1 / 2} \omega(n, y)^{1 / 2} \omega(n, z)^{1 / 2} .
\end{gathered}
$$

By (5.2) we have that this is symmetric in $m$ and $n$, hence $S_{m} S_{m}^{*} S_{n} S_{n}^{*}=S_{n} S_{n}^{*} S_{m} S_{m}^{*}$. The result then follows from (5.1). 


\section{The isomorphism.}

We shall now employ the conclusions of (5.3) in order to construct a covariant representation of the interaction group $(C(X), G, V)$ in $C^{*}(\mathcal{G})$, which will lead to an isomorphism between the crossed product $C(X) \rtimes_{V} G$ and $C^{*}(\mathcal{G})$. As always, we keep (4.1) in force.

Consider the canonical representation

$$
\pi: C(X) \rightarrow C^{*}(\mathcal{G})
$$

given by

$$
\pi(f)(x, g, y)=f(x)[g=1][x=y], \quad \forall f \in C(X), \quad \forall(x, g, y) \in \mathcal{G} .
$$

6.1. Proposition. The pair $(\pi, \sigma)$ is a covariant representation of the interaction group $(C(X), G, V)$ in $C^{*}(\mathcal{G})$.

Proof. We are required to show that

$$
\sigma_{g} \pi(f) \sigma_{g^{-1}}=\pi\left(V_{g}(f)\right) \sigma_{g} \sigma_{g^{-1}}, \quad \forall f \in C(X), \quad \forall g \in G
$$

Case 1: Suppose that $g=n \in P$. Then, for every $(x, g, y) \in \mathcal{G}$, we have

$$
\begin{gathered}
\left.\sigma_{n} \pi(f) \sigma_{n^{-1}}\right|_{(x, g, y)}=\sum_{(x, h, z) \in \mathcal{G}} S_{n}(x, h, z) f(z) S_{n}^{*}\left(z, h^{-1} g, y\right)= \\
=\sum_{(x, h, z) \in \mathcal{G}} \omega(n, x)^{1 / 2}[h=n]\left[\theta_{n}(x)=z\right] f(z) \omega(n, y)^{1 / 2}\left[h^{-1} g=n^{-1}\right]\left[\theta_{n}(y)=z\right]= \\
=\omega(n, x)^{1 / 2} f\left(\theta_{n}(x)\right) \omega(n, y)^{1 / 2}[g=1]\left[\theta_{n}(y)=\theta_{n}(x)\right]= \\
=\left.f\left(\theta_{n}(x)\right)\left(S_{n} S_{n}^{*}\right)\right|_{(x, g, y)}=\left.\left(\pi\left(V_{n}(f)\right) \sigma_{n} \sigma_{n^{-1}}\right)\right|_{(x, g, y)},
\end{gathered}
$$

thus proving (6.1.1).

Case 2: Suppose that $g=n^{-1}$, where $n \in P$. Then, for every $(x, g, y) \in \mathcal{G}$, we have

$$
\begin{gathered}
\left.\sigma_{n^{-1}} \pi(f) \sigma_{n}\right|_{(x, g, y)}=\sum_{(x, h, z) \in \mathcal{G}} S_{n}^{*}(x, h, z) f(z) S_{n}\left(z, h^{-1} g, y\right)= \\
=\sum_{(x, h, z) \in \mathcal{G}} \omega(n, z)^{1 / 2}\left[h=n^{-1}\right]\left[\theta_{n}(z)=x\right] f(z) \omega(n, z)^{1 / 2}\left[h^{-1} g=n\right]\left[\theta_{n}(z)=y\right]= \\
=[g=1][x=y] \sum_{z \in C_{x}^{n}} \omega(n, z) f(z)=\left.[g=1][x=y] L_{n}(f)\right|_{x},
\end{gathered}
$$


so

$$
\sigma_{n^{-1}} \pi(f) \sigma_{n}=\pi\left(L_{n}(f)\right)=\pi\left(V_{n^{-1}}(f)\right) \sigma_{n^{-1}} \sigma_{n}
$$

because $\sigma_{n}$ is an isometry.

CAse 3: For a general $g \in G$, write $g=n^{-1} m$, where $n, m \in P$. By (5.3) we have $\sigma_{g}=\sigma_{n^{-1}} \sigma_{m}$, while $(2.8)$ gives $V_{g}=V_{n^{-1}} V_{m}$. Therefore

$$
\sigma_{g} \pi(f) \sigma_{g^{-1}}=\sigma_{n^{-1}} \sigma_{m} \pi(f) \sigma_{m^{-1}} \sigma_{n}=\sigma_{n^{-1}} \pi\left(V_{m}(f)\right) \sigma_{m} \sigma_{m^{-1}} \sigma_{n}=\ldots
$$

Observe that

$$
\sigma_{m} \sigma_{m^{-1}} \sigma_{n}=\sigma_{m} \sigma_{m^{-1}} \sigma_{n} \sigma_{n^{-1}} \sigma_{n}=\sigma_{n} \sigma_{n^{-1}} \sigma_{m} \sigma_{m^{-1}} \sigma_{n}
$$

so the above equals

$$
\begin{gathered}
\ldots=\sigma_{n^{-1}} \pi\left(V_{m}(f)\right) \sigma_{n} \sigma_{n^{-1}} \sigma_{m} \sigma_{m^{-1}} \sigma_{n}=\pi\left(V_{n^{-1}}\left(V_{m}(f)\right)\right) \sigma_{n^{-1}} \sigma_{n} \sigma_{n^{-1}} \sigma_{m} \sigma_{m^{-1}} \sigma_{n}= \\
=\pi\left(V_{g}(f)\right) \sigma_{n^{-1}} \sigma_{m} \sigma_{m^{-1}} \sigma_{n}=\pi\left(V_{g}(f)\right) \sigma_{g} \sigma_{g^{-1}}
\end{gathered}
$$

Given the covariant representation $(\pi, \sigma)$ above we may use [6:5.3] to define a *_ homomorphism

$$
\widehat{\pi \times} \sigma: \mathcal{T}(C(X), G, V) \rightarrow C^{*}(\mathcal{G})
$$

from the Toeplitz algebra $\mathcal{T}(C(X), G, V)$ of our interaction group [6:5.1] to the groupoid $\mathrm{C}^{*}$-algebra $C^{*}(\mathcal{G})$. Since $C(X) \rtimes_{V} G$ is the quotient of the Toeplitz algebra by the redundancy ideal $[6: 6.2]$, we need to show that $\widehat{\pi \times} \sigma$ vanishes on redundancies if we are to reach our goal of obtaining a homomorphism

$$
\pi \times \sigma: C(X) \rtimes_{V} G \rightarrow C^{*}(\mathcal{G}) .
$$

In the terminology of $[\mathbf{6}: 6.3]$ we must prove that $(\pi, \sigma)$ is strongly covariant. The following two Lemmas will be later used to further this goal.

But first let us introduce some notation. By a word in $G$ we shall mean any finite sequence $\alpha=\left(g_{1}, \ldots, g_{k}\right)$, where $g_{i} \in G$. If $\sigma$ is a partial representation of $G$ we shall let

$$
\sigma_{\alpha}=\sigma_{g_{1}} \ldots \sigma_{g_{n}}
$$

6.2. Lemma. Let $\sigma$ be a partial representation of $G$ in some $C^{*}$-algebra $B$ such that $\sigma_{n}$ is an isometry for every $n \in P$. Then for every word $\alpha$ in $G$ there exists another word $\beta$, such that

$$
\sigma_{\alpha} \sigma_{\beta}=\sigma_{n}
$$

for some $n \in P$. 
Proof. Letting $\alpha=\left(g_{1}, \ldots, g_{k}\right)$ we shall prove the statement by induction on $k$. If $k=1$, in which case $\alpha=\left(g_{1}\right)$, write $g_{1}=n m^{-1}$, with $n, m \in P$, and let $\beta=(m)$. Then

$$
\sigma_{\alpha} \sigma_{\beta}=\sigma_{g_{1}} \sigma_{m}=\sigma_{g_{1}} \sigma_{m} \sigma_{m}^{*} \sigma_{m}=\sigma_{g_{1} m} \sigma_{m}^{*} \sigma_{m}=\sigma_{n}
$$

thus proving the result for $k=1$.

If $k \geq 2$, let $\alpha^{\prime}=\left(g_{2}, \ldots, g_{k}\right)$ and use induction to get $\beta^{\prime}$ and $n^{\prime}$ such that $\sigma_{\alpha^{\prime}} \sigma_{\beta^{\prime}}=\sigma_{n^{\prime}}$. Then

$$
\sigma_{\alpha} \sigma_{\beta^{\prime}}=\sigma_{g_{1}} \sigma_{\alpha^{\prime}} \sigma_{\beta^{\prime}}=\sigma_{g_{1}} \sigma_{n^{\prime}}=\sigma_{g_{1}} \sigma_{n^{\prime}} \sigma_{n^{\prime}}^{*} \sigma_{n^{\prime}}=\sigma_{g_{1} n^{\prime}} \sigma_{n^{\prime}}^{*} \sigma_{n^{\prime}}=\sigma_{g_{1} n^{\prime}},
$$

and the conclusion follows from the case $k=1$.

The following idea has already been used in several occasions, e.g. in $[7: 8.6,7.2]$.

6.3. Lemma. Given $n \in P$ there exists a finite set $\left\{u_{1}, \ldots, u_{n}\right\} \subseteq C(X)$ such that

$$
\sum_{i=1}^{n} \pi\left(u_{i}\right) S_{n} S_{n}^{*} \pi\left(u_{i}\right)=1 .
$$

Proof. Let $\left\{V_{i}\right\}_{i=1}^{n}$ be a finite open cover of $X$ such that $\theta_{n}$ is injective on each $V_{i}$ and let $\left\{\phi_{i}\right\}_{i=1}^{n}$ be a partition of unity subordinated to $\left\{V_{i}\right\}_{i=1}^{n}$. Define $u_{i}(x)=\phi_{i}(x) \omega(n, x)^{-1 / 2}$ and observe that for every $(x, g, y) \in \mathcal{G}$ we have, using (5.3.1), that

$$
\begin{gathered}
\left.\sum_{i=1}^{n} \pi\left(u_{i}\right) S_{n} S_{n}^{*} \pi\left(u_{i}\right)\right|_{(x, g, y)}=\left.\sum_{i=1}^{n} u_{i}(x) u_{i}(y) S_{n} S_{n}^{*}\right|_{(x, g, y)}= \\
=\sum_{i=1}^{n} u_{i}(x) u_{i}(y) \omega(n, x)^{1 / 2} \omega(n, y)^{1 / 2}[g=1]\left[\theta_{n}(x)=\theta_{n}(y)\right]= \\
=\sum_{i=1}^{n} u_{i}(x)^{2} \omega(n, x)[g=1][x=y]=\sum_{i=1}^{n} \phi_{i}(x)[g=1][x=y]=[g=1][x=y],
\end{gathered}
$$

proving the statement.

We now may prove the following crucial technical result:

6.4. Proposition. The representation $(\pi, \sigma)$ of $(C(X), G, V)$ given by (6.1) is strongly covariant.

Proof. Recall that to say that $(\pi, \sigma)$ is strongly covariant is to say that $\widehat{\pi \times} \sigma$ vanishes on all redundancies [6:6.3]. In order to shorten our notation we will write $\widehat{\rho}=\widehat{\pi \times} \sigma$.

Let $\alpha$ be a word in $G$ and let $k$ be an $\alpha$-redundancy. Then $k \mathcal{M}_{\alpha}=0$, so

$$
0=\widehat{\rho}\left(k \mathcal{M}_{\alpha}\right)=\widehat{\rho}(k) \widehat{\rho}\left(\mathcal{M}_{\alpha}\right)=\widehat{\rho}(k) \pi(C(X)) \sigma_{\alpha} \pi(C(X))
$$


and in particular $\hat{\rho}(k) \pi(C(X)) \sigma_{\alpha}=0$. Using Lemma (6.2) choose a word $\beta$ such that $\sigma_{\alpha} \sigma_{\beta}=\sigma_{n}$, for some $n \in P$, so

$$
0=\widehat{\rho}(k) \pi(C(X)) \sigma_{\alpha} \sigma_{\beta}=\widehat{\rho}(k) \pi(C(X)) \sigma_{n} .
$$

Recalling that $\sigma_{n}=S_{n}$ and using (6.3) we have that

$$
\widehat{\rho}(k)=\sum_{i=1}^{n} \widehat{\rho}(k) \pi\left(u_{i}\right) S_{n} S_{n}^{*} \pi\left(u_{i}\right)=0 .
$$

Since $\widehat{\pi \times} \sigma$ vanishes on redundancies it factors through the quotient of $\mathcal{T}(C(X), G, V)$ by the redundancy ideal and hence defines a *-homomorphism

$$
\pi \times \sigma: C(X) \rtimes_{V} G \rightarrow C^{*}(\mathcal{G}) .
$$

Our next major goal will be to prove that $\pi \times \sigma$ is an isomorphism. The proof of injectivity will be based on $[\mathbf{6}: 10.6]$ so we are required to first verify the following:

6.5. Proposition. The covariant representation $(\pi, \sigma)$ of $(C(X), G, V)$ given by (6.1) is non-degenerate.

Proof. Recall that to say that $(\pi, \sigma)$ is non-degenerate is to say that the map

$$
a \in C(X) \mapsto \pi(a) \sigma_{\alpha} \in C^{*}(\mathcal{G})
$$

is injective for every word $\alpha$ in $G$. So suppose that $a \in C(X)$ is such that $\pi(a) \sigma_{\alpha}=0$. Then, picking $\beta$ and $n$ as in (6.2) we have

$$
0=\pi(a) \sigma_{\alpha} \sigma_{\beta}=\pi(a) \sigma_{n}=\pi(a) S_{n} .
$$

Given $x \in X$ consider the element $\left(x, n, \theta_{n}(x)\right) \in \mathcal{G}$. Thinking of $\pi(a) S_{n}$ as a compactly supported function on $\mathcal{G}$ we compute

$$
0=\left.\pi(a) \sigma_{n}\right|_{\left(x, n, \theta_{n}(x)\right)}=a(x) \omega(n, x)^{1 / 2},
$$

so it follows that $a=0$.

Notice that we have used that $\omega$ never vanishes in the proof above. The following is the main result of this section:

6.6. Theorem. In addition to the hypotheses of (4.1) suppose that $G$ is amenable and let $(C(X), G, V)$ be the interaction group provided by (2.8), namely, if $g=n^{-1} m$, with $n, m \in P$,

$$
\left.V_{g}(f)\right|_{y}=\sum_{\theta_{n}(x)=y} \omega(n, x) f\left(\theta_{m}(x)\right), \quad \forall f \in C(X), \quad \forall y \in X .
$$

Moreover consider the strongly covariant representation $(\pi, \sigma)$ of $(C(X), G, V)$ in the groupoid $C^{*}$-algebra of $\mathcal{G}$ given by (6.1) and (6.4). Then

$$
\pi \times \sigma: C(X) \rtimes_{V} G \rightarrow C^{*}(\mathcal{G})
$$

is an isomorphism. 
Proof. By (6.5) and [6:10.6] we have that $\pi \times \sigma$ is injective on each $C_{g}$, where $\left\{C_{g}\right\}_{g \in G}$ is the grading of $C(X) \rtimes_{V} G$ given by $[6: 7.2]$.

Observe that $C^{*}(\mathcal{G})$ also admits a grading $\left\{D_{g}\right\}_{g \in G}$ such that $\pi(f) \in D_{1}$, for all $f \in C(X)$, and $S_{n} \in D_{n}$, for every $n \in P$. If follows that $\pi \times \sigma$ is a graded homomorphism in the sense that $(\pi \times \sigma)\left(C_{g}\right) \subseteq D_{g}$, for every $g \in G$. Let

$$
\begin{array}{lccc}
E_{1}: & C(X) \rtimes_{V} G & \rightarrow & C_{1} \\
E_{2}: & C^{*}(\mathcal{G}) & \rightarrow & D_{1}
\end{array}
$$

be the associated conditional expectations, so we have that

$$
E_{2}((\pi \times \sigma)(a))=(\pi \times \sigma)\left(E_{1}(a)\right), \quad \forall a \in C(X) \rtimes_{V} G .
$$

If $a$ is such that $(\pi \times \sigma)(a)=0$, then

$$
(\pi \times \sigma)\left(E_{1}\left(a^{*} a\right)\right)=E_{2}\left((\pi \times \sigma)\left(a^{*} a\right)\right)=0 .
$$

Since $E_{1}\left(a^{*} a\right) \in C_{1}$, and $\pi \times \sigma$ is injective on $C_{1}$, as observed above, we have that $E_{1}\left(a^{*} a\right)=$ 0 . Given that $G$ is amenable, the Fell bundle $\left\{C_{g}\right\}_{g \in G}$ must also be amenable [4:4.7] and hence $E_{1}$ is faithful by [4:2.12]. So $a=0$.

In order to prove that $\pi \times \sigma$ is surjective observe that the range of $\pi \times \sigma$ contains $\pi(f)$ and $S_{n}$, for every $f \in C(X)$ and $n \in P$, so surjectivity follows from (4.5).

\section{Lattice-ordered semigroups.}

The reader might have a few examples in mind of semigroups acting on compact spaces. But before we can apply (2.8), or any of our results based on it, we need to provide a normalized coherent cocycle, a task which might not be entirely trivial.

In what follows we plan to show that coherent cocycles are often present in a number of situations. With this goal in mind we will now study some elementary properties of semigroups which will later play an important role in providing applications of our results.

We will suppose throughout that $G$ is a group and that $P$ is a subsemigroup of $G$ such that $P \cap P^{-1}=\{1\}$. In most of our examples $G$ will be commutative but this does not seem to be too significant for the general theory. We shall therefore not suppose that $G$ is commutative here.

One may define a left-invariant order on $G$ by saying that

$$
x \leq y \Longleftrightarrow x^{-1} y \in P .
$$

7.1. Definition. We shall say that the pair $(G, P)$ is a lattice-ordered group if, for every $x$ and $y$ in $G$, the set $\{x, y\}$ admits a least upper bound $x \vee y$ and a greatest lower bound $x \wedge y$. 
From now on we will fix a lattice-ordered group $(G, P)$.

7.2. Definition. A mini-square is by definition a quadruple of elements $(s, t, u, v) \in P^{4}$ such that

(i) $s u=t v$,

(ii) $s \wedge t=1$,

(iii) $u^{-1} \vee v^{-1}=1$.

Observe that the last condition is equivalent to saying that $u \wedge v=1$, where " $\wedge$ " denotes the greatest lower bound relative to the right-invariant order induced by $P$. Since it is a bit awkward to deal with two distinct order relations at the same time we will make no further references to the right-invariant order relation.

It is interesting to represent mini-squares by a diagram such as

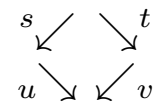

7.3. Proposition. For every $m, n \in G$ let

$$
\begin{aligned}
& s=(m \wedge n)^{-1} m, \quad t=(m \wedge n)^{-1} n, \\
& u=m^{-1}(m \vee n), \quad v=n^{-1}(m \vee n) .
\end{aligned}
$$

Then $(s, t, u, v)$ is a mini-square.

Proof. Since $(m \wedge n) \leq m, n \leq(m \vee n)$ it is obvious that $s, t, u, v \in P$. Obviously

$$
s u=(m \wedge n)^{-1}(m \vee n)=t v .
$$

In order to show that $s \wedge t=1$, let $g \in G$ be such that $g \leq s, t$. Therefore

$$
(m \wedge n) g \leq(m \wedge n) s=m, \quad \text { and } \quad(m \wedge n) g \leq(m \wedge n) t=n
$$

It follows that

$$
(m \wedge n) g \leq(m \wedge n)
$$

and hence that $g \leq e$. This shows that $s \wedge t=1$. Next we show that $u^{-1} \vee v^{-1}=1$. For this suppose that $g \geq u^{-1}, v^{-1}$. Then

$$
(m \vee n) g \geq(m \vee n) u^{-1}=m, \quad \text { and } \quad(m \vee n) g \geq(m \vee n) v^{-1}=n
$$

Therefore

$$
(m \vee n) g \geq(m \vee n)
$$

whence $g \geq e$. 
7.4. Proposition. Let $(s, t, u, v)$ be a mini-square. Then $s \vee t=s u=t v$.

Proof. By definition $s u=t v$. It is also clear that

$$
s u \geq s, \quad \text { and } \quad s u=t v \geq t .
$$

Now suppose that $g \geq s, t$. Then

$$
(s u)^{-1} g \geq(s u)^{-1} s=u^{-1}, \quad \text { and } \quad(s u)^{-1} g \geq(s u)^{-1} t=(t v)^{-1} t=v^{-1} .
$$

Therefore

$$
(s u)^{-1} g \geq u^{-1} \vee v^{-1}=1,
$$

which implies that $g \geq s u$, as desired.

7.5. Corollary. Given $s, t \in P$ such that $s \wedge t=1$, there exists a unique pair $(u, v) \in P \times P$ such that $(s, t, u, v)$ is a mini-square.

Proof. For existence apply $(7.3)$ to the pair $(m, n):=(s, t)$. For uniqueness observe that by (7.4) we have that $u=s^{-1}(s \vee t)$ and $v=t^{-1}(s \vee t)$.

Notice that if $G$ is commutative and $s, t \in G$ are such that $s \wedge t=1$, then

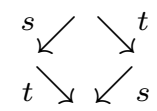

is clearly a mini-square. By (7.5) these are the only possible mini-squares.

\section{Admissible semigroup actions and cocycles.}

The main goal of this section is to present sensible conditions on semigroup actions and cocycles from which one may deduce coherence, hence providing examples of interaction groups by (2.8).

We begin by fixing a lattice-ordered group $(G, P)$. Observe that (2.8.ii) is automatically satisfied:

8.1. Proposition. If $(G, P)$ is a lattice-ordered group then $G=P^{-1} P=P P^{-1}$.

Proof. Given $x \in G$, let $y=x \wedge e$. Then $y \leq x$ and hence $n:=y^{-1} x \in P$. Observing that $y^{-1} \in P$, because $y \leq e$, we have that $x=y n \in P^{-1} P$. This shows that $G=P^{-1} P$.

Next let $n=x \vee e$. Since $x \leq n$ we have that $m:=x^{-1} n \in P$. So $x=n m^{-1} \in P P^{-1}$.

8.2. Definition. We shall say that a right action $\theta: P \rightarrow \operatorname{End}(X)$ is admissible if, given any mini-square

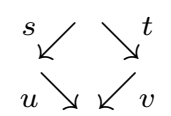

and $x, y \in X$ such that $\theta_{u}(x)=\theta_{v}(y)$, there exists a unique $z \in X$ such that $\theta_{s}(z)=x$, and $\theta_{t}(z)=y$. 
From now on we shall fix an admissible action $\theta$ of $P$ on $X$. Our first task will be to describe sets of the form $\theta_{m}^{-1}(p) \cap \theta_{n}^{-1}(q)$.

8.3. Lemma. Given $m, n \in P$ and $p, q \in X$, let $(s, t, u, v)$ be the mini-square given by (7.3) in terms of $m$ and $n$.

(i) If $\theta_{u}(p) \neq \theta_{v}(q)$, then

$$
\theta_{m}^{-1}(p) \cap \theta_{n}^{-1}(q)=\emptyset
$$

(ii) If $\theta_{u}(p)=\theta_{v}(q)$, and $w$ is the unique element in $X$ such that $\theta_{s}(w)=p$, and $\theta_{t}(w)=q$, then

$$
\theta_{m}^{-1}(p) \cap \theta_{n}^{-1}(q)=\theta_{m \wedge n}^{-1}(w) .
$$

Proof. (i) By contradiction let $x \in \theta_{m}^{-1}(p) \cap \theta_{n}^{-1}(q)$. Then

$$
\theta_{u}(p)=\theta_{u}\left(\theta_{m}(x)\right)=\theta_{m u}(x)=\theta_{n v}(x)=\theta_{v}\left(\theta_{n}(x)\right)=\theta_{v}(q),
$$

contradicting the hypothesis.

(ii) If $\theta_{m \wedge n}(x)=w$, then

$$
\theta_{m}(x)=\theta_{(m \wedge n) s}(x)=\theta_{s}\left(\theta_{m \wedge n}(x)\right)=\theta_{s}(w)=p,
$$

and similarly $\theta_{n}(x)=q$. Conversely, if $\theta_{m}(x)=p$, and $\theta_{n}(x)=q$, set $w^{\prime}=\theta_{m \wedge n}(x)$. Then

$$
\theta_{s}\left(w^{\prime}\right)=\theta_{s}\left(\theta_{m \wedge n}(x)\right)=\theta_{(m \wedge n) s}(x)=\theta_{m}(x)=p,
$$

and similarly $\theta_{t}\left(w^{\prime}\right)=q$. By uniqueness we have that $w^{\prime}=w$, so $\theta_{m \wedge n}(x)=w$, and hence $x \in \theta_{m \wedge n}^{-1}(w)$.

8.4. Proposition. Let $\bar{z} \in X$, let $(s, t, u, v)$ be a mini-square, and put $\bar{x}=\theta_{s}(\bar{z})$ and $\bar{y}=\theta_{t}(\bar{z})$. Then the map

given by

$$
\phi: C_{\bar{z}}^{s \vee t} \rightarrow C_{\bar{x}}^{u} \times C_{\bar{y}}^{v}
$$

$$
\phi(z)=\left(\theta_{s}(z), \theta_{t}(z)\right)
$$

is a bijection.

Proof. Given $z \in C_{\bar{z}}^{s \vee t}$, let $x=\theta_{s}(z)$ and $y=\theta_{t}(z)$. Then, recalling from (7.4) that $s \vee t=s u=t v$, notice that

$$
\theta_{u}(x)=\theta_{u}\left(\theta_{s}(z)\right)=\theta_{s u}(z)=\theta_{s \vee t}(z)=\theta_{s \vee t}(\bar{z})=\theta_{s u}(\bar{z})=\theta_{u}\left(\theta_{s}(\bar{z})\right)=\theta_{u}(\bar{x}),
$$

proving that $x \in C_{\bar{x}}^{u}$. Similarly one shows that $y \in C_{\bar{y}}^{v}$, so $\phi(z)=(x, y)$ indeed lies in $C_{\bar{x}}^{u} \times C_{\bar{y}}^{v}$

$$
\begin{aligned}
\text { Let }(x, y) & \in C_{\bar{x}}^{u} \times C_{\bar{y}}^{v} \text {. Then } \\
\theta_{u}(x) & =\theta_{u}(\bar{x})=\theta_{u}\left(\theta_{s}(\bar{z})\right)=\theta_{s u}(\bar{z})=\theta_{t v}(\bar{z})=\theta_{v}\left(\theta_{t}(\bar{z})\right)=\theta_{v}(\bar{y})=\theta_{v}(y) .
\end{aligned}
$$

By hypothesis there exists a unique $z \in X$ such that $\phi(z)=(x, y)$. We claim that $z$ must in fact lie in $C_{\bar{z}}^{s \vee t}$. To see this notice that

$$
\theta_{s \vee t}(z)=\theta_{s u}(z)=\theta_{u}\left(\theta_{s}(z)\right)=\theta_{u}(x)=\theta_{s u}(\bar{z})=\theta_{s \vee t}(\bar{z}) .
$$

This shows that $\phi$ is bijective. 
Let us now suppose we are given a normalized cocycle $\omega$. Given $x \in X$ and $n \in P$ consider the restriction of $\omega(n, \cdot)$ to $C_{x}^{n}$. By $(2.1)$ we may view this as a probability distribution on $C_{x}^{n}$.

Under the hypotheses of (8.4) observe that we then have two probability distributions on $C_{\bar{z}}^{s \vee t}$, namely $\omega(s \vee t, \cdot)$ on the one hand, and the product distribution of $\omega(u, \cdot)$ and $\omega(v, \cdot)$ on the other.

Our next result touches upon the question as to whether these probability distributions coincide.

8.5. Proposition. Given a cocycle $\omega$, consider the following statements:

(i) for every $z \in X$ and every mini-square $(s, t, u, v)$ one has that $\omega(s \vee t, z)=$ $\omega\left(u, \theta_{s}(z)\right) \omega\left(v, \theta_{t}(z)\right)$.

(ii) for every $z \in X$ and every mini-square $(s, t, u, v)$ one has that $\omega(t, z)=\omega\left(u, \theta_{s}(z)\right)$,

(iii) for every $s, t \in P$ with $s \wedge t=1$, and for every $z \in X$ one has that $\omega(s \vee t, z)=$ $\omega(s, z) \omega(t, z)$.

Then (i) $\Leftarrow$ (ii) $\Rightarrow$ (iii). If $\omega$ never vanishes then also (i) $\Rightarrow$ (ii) $\Leftarrow$ (iii).

Proof.

(ii) $\Rightarrow($ i). We have

$$
\omega(s \vee t, z)=\omega(t v, z)=\omega(t, z) \omega\left(v, \theta_{t}(z)\right)=\omega\left(u, \theta_{s}(z)\right) \omega\left(v, \theta_{t}(z)\right)
$$

(ii) $\Rightarrow$ (iii). Given $(s, t)$ as in (iii) pick $u$ and $v$ such that $(s, t, u, v)$ is a mini-square by (7.5). We then have

$$
\omega(s \vee t, z)=\omega(s u, z)=\omega(s, z) \omega\left(u, \theta_{s}(z)\right)=\omega(s, z) \omega(t, z)
$$

(i) $\Rightarrow$ (ii). One has

$$
\omega\left(u, \theta_{s}(z)\right) \omega\left(v, \theta_{t}(z)\right)=\omega(s \vee t, z)=\omega(t v, z)=\omega(t, z) \omega\left(v, \theta_{t}(z)\right)
$$

Since $\omega\left(v, \theta_{t}(z)\right) \neq 0$, we have that $\omega\left(u, \theta_{s}(z)\right)=\omega(t, z)$.

(iii) $\Rightarrow$ (ii). We have

$$
\omega(s, z) \omega(t, z)=\omega(s \vee t, z)=\omega(s u, z)=\omega(s, z) \omega\left(u, \theta_{s}(z)\right) .
$$

Since $\omega(s, z) \neq 0$ we conclude that $\omega(t, z)=\omega\left(u, \theta_{s}(z)\right)$.

We therefore make the following:

8.6. Definition. A cocycle $\omega$ is said to be admissible if (8.5.ii) and hence also (8.5.i \& iii) holds. 
The reason why we are interested in admissible cocycles is given below.

8.7. Proposition. If $\theta$ and $\omega$ are admissible then $\omega$ is coherent.

Proof. We first claim that if $\theta_{m \vee n}(z) \neq \theta_{m \vee n}(x)$, then both sides of (2.7) vanish. By symmetry it is enough to show that this is so for the right-hand side of (2.7).

Let $(s, t, u, v)$ be the mini-square obtained from (7.3) from $m$ and $n$, and set $p=\theta_{m}(z)$, and $q=\theta_{n}(x)$. Then

$$
\theta_{u}(p)=\theta_{u}\left(\theta_{m}(z)\right)=\theta_{m u}(z)=\theta_{m \vee n}(z) \neq \theta_{m \vee n}(x)=\theta_{n v}(x)=\theta_{v}\left(\theta_{n}(x)\right)=\theta_{v}(q) .
$$

That is, $\theta_{u}(p) \neq \theta_{v}(q)$, and hence by (8.3) we have that

$$
C_{x}^{n} \cap C_{z}^{m}=\theta_{n}^{-1}(q) \cap \theta_{m}^{-1}(p)=\emptyset
$$

The right-hand side of (2.7) then vanishes because $C_{x, z}^{n, m}=\emptyset$.

Suppose now that $\theta_{m \vee n}(z)=\theta_{m \vee n}(x)$. Letting $(s, t, u, v), p$ and $q$ be as above we than conclude similarly that $\theta_{u}(p)=\theta_{v}(q)$. Again by (8.3) we have that

$$
C_{x}^{n} \cap C_{z}^{m}=\theta_{n}^{-1}(q) \cap \theta_{m}^{-1}(p)=\theta_{m \wedge n}^{-1}(w),
$$

where $w$ is the unique element in $X$ such that $\theta_{s}(w)=p$, and $\theta_{t}(w)=q$. Therefore the right-hand side of (2.7) satisfies

$$
\begin{gathered}
\sum_{y \in C_{z}^{m} \cap C_{x}^{n}} \omega(m, y) \omega(n, x)=\sum_{y \in \theta_{m \wedge n}^{-1}(w)} \omega((m \wedge n) s, y) \omega(n, x)= \\
=\sum_{y \in \theta_{m \wedge n}^{-1}(w)} \omega(m \wedge n, y) \omega\left(s, \theta_{m \wedge n}(y)\right) \omega(n, x)= \\
=\left(\sum_{y \in \theta_{m \wedge n}^{-1}(w)} \omega(m \wedge n, y)\right) \omega(s, w) \omega(n, x)=\omega(s, w) \omega(n, x)=(\star) .
\end{gathered}
$$

Observe that by symmetry $(t, s, v, u)$ is a mini-square as well. Thus, applying (8.5.ii), we have that

$$
\omega(s, w) \stackrel{(8.5 . i i)}{=} \omega\left(v, \theta_{t}(w)\right)=\omega\left(v, \theta_{n}(x)\right) .
$$

Therefore

$$
(\star)=\omega\left(v, \theta_{n}(x)\right) \omega(n, x)=\omega(n v, x)=\omega(m \vee n, x) .
$$

Having arrived at an expression which is symmetric in the variables $m$ and $n$, the proof is complete.

We thus arrive at the following important result: 
8.8. Corollary. Let $(G, P)$ be a lattice-ordered group, let $\theta$ be an admissible right action of $P$ on a compact space $X$, and let $\omega$ be an admissible normalized cocycle. Then there exists a unique interaction group $V=\left\{V_{g}\right\}_{g \in G}$ on $C(X)$, such that, if $g=n^{-1} m$, with $n, m \in P$, then

$$
\left.V_{g}(f)\right|_{y}=\sum_{\theta_{n}(x)=y} \omega(n, x) f\left(\theta_{m}(x)\right), \quad \forall f \in C(X), \quad \forall y \in X
$$

Proof. Follows immediately from the above result plus (8.1) and (2.8).

\section{Example: single endomorphism.}

Beginning with this section we shall give several examples of admissible, therefore coherent, cocycles for which one may apply (6.6).

Let $G=\mathbb{Z}$ and let $P=\mathbb{N}$. It is obvious that $(\mathbb{Z}, \mathbb{N})$ is a lattice-ordered group. Given any endomorphism $T$ of $X$ define an action $\theta$ of $\mathbb{N}$ on $X$ by

$$
\theta: n \in \mathbb{N} \mapsto T^{n} \in \operatorname{End}(X)
$$

The only mini-squares in $\mathbb{N}$ are of the form

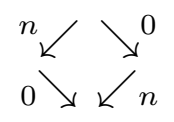

where $n \in \mathbb{N}$, and its reflections across the vertical axis, and hence it is obvious that $\theta$ is admissible.

For each $x \in X$, let

$$
\omega(0, x)=1, \quad \text { and } \quad \omega(1, x)=\frac{1}{\left|C_{x}^{1}\right|}
$$

For all $n \geq 1$, define $\omega(n+1, x)$ recursively by

$$
\omega(n+1, x)=\omega(n, x) \omega\left(1, \theta_{n}(x)\right) .
$$

It is then easy to prove by induction that $\omega$ is a normalized admissible cocycle which does not vanish anywhere.

We should observe that, in the case of the present example, Theorem (6.6) was already proved in $[7: 9.1]$. 


\section{Example: star-commuting endomorphisms.}

This example is inspired by $[\mathbf{1}]$.

10.1. Definition. Let $S$ and $T$ be commuting maps on a set $X$. We shall say that the pair $(S, T)$ star-commutes $[\mathbf{1}]$ if for every $x, y \in X$ such that $T(x)=S(y)$, there exists a unique $z \in X$ such that $S(z)=x$, and $T(z)=y$.

Consider $G=\mathbb{Z} \times \mathbb{Z}$ and let $P=\mathbb{N} \times \mathbb{N}$. It is obvious that $(\mathbb{Z} \times \mathbb{Z}, \mathbb{N} \times \mathbb{N})$ is a lattice-ordered group with

$$
\left(n_{1}, m_{1}\right) \vee\left(n_{2}, m_{2}\right)=\left(\max \left\{n_{1}, n_{2}\right\}, \max \left\{m_{1}, m_{2}\right\}\right)
$$

and

$$
\left(n_{1}, m_{1}\right) \wedge\left(n_{2}, m_{2}\right)=\left(\min \left\{n_{1}, n_{2}\right\}, \min \left\{m_{1}, m_{2}\right\}\right) .
$$

Observe that all mini-squares are either given by

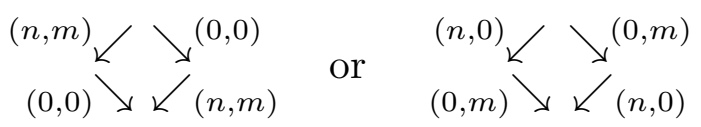

where $n, m \in \mathbb{N}$, or the reflections of the above across a vertical axis.

Let $S$ and $T$ be commuting endomorphisms of a compact Hausdorff space $X$ and define

$$
\theta:(n, m) \in \mathbb{N} \times \mathbb{N} \mapsto S^{n} T^{m} \in \operatorname{End}(X)
$$

It is clear that $\theta$ is an action of $\mathbb{N} \times \mathbb{N}$ on $X$. Observe that (10.1) is then equivalent to saying that the condition of (8.2) holds for the special mini-square

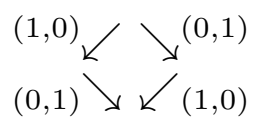

10.2. Proposition. If $(S, T)$ star-commutes then $\theta$ is admissible.

Proof. Leaving aside the trivial mini-squares (i.e., those involving the trivial group element) and taking advantage of vertical symmetry, we consider only mini-squares of the form

$$
\begin{aligned}
& (n, 0) \swarrow \searrow^{(0, m)} \\
& (0, m) \searrow \swarrow(n, 0)
\end{aligned}
$$

where $n, m \geq 1$. Our task therefore consists in showing the following:

"If $T^{m}(x)=S^{n}(y)$, then there exists a unique $z \in X$ such that $S^{n}(z)=x$, and $T^{m}(z)=y . "$

We shall prove this by induction on $n+m$. Given that $n, m \geq 1$, we see that the lowest possible value for $n+m$ is 2 , in which case the claim follows directly from the hypothesis. 
Assuming $n+m \geq 3$, suppose without lack of generality that $n \geq 2$. Then, letting $y^{\prime}=S(y)$, notice that $T^{m}(x)=S^{n-1}\left(y^{\prime}\right)$, so we have by the induction hypothesis that there exists a unique $w \in X$ such that

$$
S^{n-1}(w)=x, \quad \text { and } \quad T^{m}(w)=y^{\prime} .
$$

Next, observing that $T^{m}(w)=y^{\prime}=S(y)$, and that $1+m<n+m$, we see, again by induction, that there exists $z \in X$ such that

$$
S(z)=w, \quad \text { and } \quad T^{m}(z)=y
$$

Clearly

$$
S^{n}(z)=S^{n-1}(S(z))=S^{n-1}(w)=x .
$$

Thus $z$ solves our existence question. As for uniqueness assume that $z^{\prime}$ is such that $S^{n}\left(z^{\prime}\right)=x$, and $T^{m}\left(z^{\prime}\right)=y$. Setting $w^{\prime}=S\left(z^{\prime}\right)$, we have

$$
S^{n-1}\left(w^{\prime}\right)=S^{n-1}\left(S\left(z^{\prime}\right)\right)=S^{n}\left(z^{\prime}\right)=x
$$

and

$$
T^{m}\left(w^{\prime}\right)=T^{m}\left(S\left(z^{\prime}\right)\right)=S\left(T^{m}\left(z^{\prime}\right)\right)=S(y)=y^{\prime} .
$$

Comparing the above with $(\dagger)$, and using the uniqueness part of the induction hypothesis, we see that necessarily $w^{\prime}=w$. Observing that

$$
S\left(z^{\prime}\right)=w, \quad \text { and } \quad T^{m}\left(z^{\prime}\right)=y
$$

we then conclude, in view of $(\ddagger)$ and the induction hypothesis, that $z^{\prime}=z$.

We next construct an admissible hence coherent cocycle. Let

$$
\omega_{S}, \omega_{T}: \mathbb{N} \times X \rightarrow \mathbb{R}_{+}
$$

be each given as in section (9) relatively to $S$ and $T$, respectively.

10.3. Lemma. If $(S, T)$ star-commutes then for every $n \in \mathbb{N}$ and every $x \in X$ one has

$$
\omega_{S}(n, x)=\omega_{S}(n, T(x)), \quad \text { and } \quad \omega_{T}(n, x)=\omega_{T}(n, S(x))
$$

Proof. We prove the first assertion only, doing so by induction on $n$. Obviously it holds for $n=0$. Speaking of the case $n=1$, let $x \in X$. Adopting the notation

$$
C_{x}^{S}=\left\{x^{\prime} \in X: S\left(x^{\prime}\right)=S(x)\right\}
$$

consider the map

$$
\phi: x^{\prime} \in C_{x}^{S} \mapsto T\left(x^{\prime}\right) \in C_{T(x)}^{S}
$$


That $T\left(x^{\prime}\right)$ in fact belongs to $C_{T(x)}^{S}$ follows from

$$
S\left(T\left(x^{\prime}\right)\right)=T\left(S\left(x^{\prime}\right)\right)=T(S(x))=S(T(x)) .
$$

We claim that this map is bijective. In order to show that it is one-to-one let $x^{\prime}, x^{\prime \prime} \in C_{x}^{S}$ be such that $T\left(x^{\prime}\right)=T\left(x^{\prime \prime}\right)$. Consider the diagram

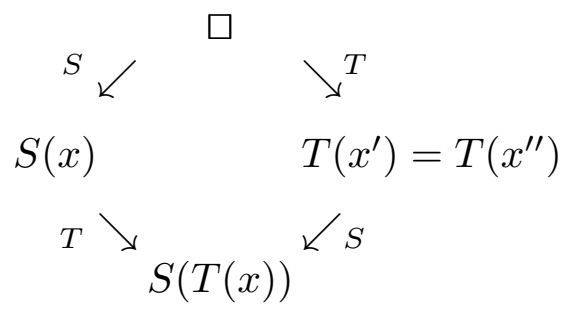

Since both $x^{\prime}$ and $x^{\prime \prime}$ fit in the box we conclude that $x^{\prime}=x^{\prime \prime}$, by the fact that $(S, T)$ star-commutes.

In order to show surjectivity let $y \in C_{T(x)}^{S}$ and consider the diagram

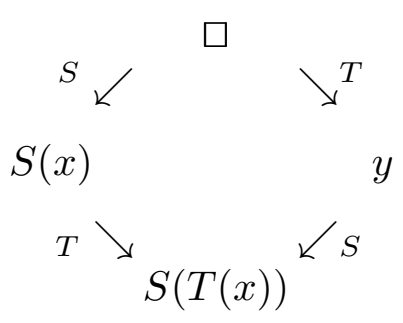

By hypothesis there exists a $x^{\prime}$ which fits in the box. Obviously such a $x^{\prime}$ lies in $C_{x}^{S}$ and $T\left(x^{\prime}\right)=y$. This proves that our map is in fact bijective.

As a consequence we have that $\left|C_{x}^{S}\right|=\left|C_{T(x)}^{S}\right|$, so that

$$
\omega_{S}(1, x)=\frac{1}{\left|C_{x}^{S}\right|}=\frac{1}{\left|C_{T(x)}^{S}\right|}=\omega_{S}(1, T(x)) .
$$

Given $n \geq 1$ we have by induction that

$$
\begin{gathered}
\omega_{S}(n+1, T(x))=\omega_{S}(n, T(x)) \omega_{S}\left(1, S^{n}(T(x))\right)=\omega_{S}(n, x) \omega_{S}\left(1, T\left(S^{n}(x)\right)\right)= \\
=\omega_{S}(n, x) \omega_{S}\left(1, S^{n}(x)\right)=\omega_{S}(n+1, x) .
\end{gathered}
$$

We should observe that the cocycles $\omega_{S}$ and $\omega_{T}$ given by section (9) are certainly not unique. Nevertheless, if a different choice of $\omega_{S}$ and $\omega_{T}$ was made in the above proof it is not clear that we could carry it on. It is therefore interesting to pinpoint exactly to what extent are those cocycles special. 
10.4. Theorem. If $(S, T)$ star-commutes then the map $\omega_{T}:(\mathbb{N} \times \mathbb{N}) \times X \rightarrow \mathbb{R}_{+}$, defined by

$$
\omega((n, m), x)=\omega_{S}(n, x) \omega_{T}(m, x)
$$

is a normalized admissible cocycle which does not vanish anywhere.

Proof. It is obvious that $\omega((0,0), x)=1$, for all $x$, so let us check the cocycle identity (2.2). Given $n=\left(n_{1}, n_{2}\right)$ and $m=\left(m_{1}, m_{2}\right)$ in $\mathbb{N} \times \mathbb{N}$ we have

$$
\begin{gathered}
\omega(n+m, x)=\omega\left(\left(n_{1}+m_{1}, n_{2}+m_{2}\right), x\right)=\omega_{S}\left(n_{1}+m_{1}, x\right) \omega_{T}\left(n_{2}+m_{2}, x\right)= \\
=\omega_{S}\left(n_{1}, x\right) \omega_{S}\left(m_{1}, S^{n_{1}}(x)\right) \omega_{T}\left(n_{2}, x\right) \omega_{T}\left(m_{2}, T^{n_{2}}(x)\right)= \\
\stackrel{(10.3)}{=} \omega_{S}\left(n_{1}, x\right) \omega_{S}\left(m_{1}, T^{n_{2}} S^{n_{1}}(x)\right) \omega_{T}\left(n_{2}, x\right) \omega_{T}\left(m_{2}, S^{n_{1}} T^{n_{2}}(x)\right)= \\
=\omega\left(\left(n_{1}, n_{2}\right), x\right) \omega\left(\left(m_{1}, m_{2}\right), S^{n_{1}} T^{n_{2}}(x)\right)=\omega(n, x) \omega\left(m, \theta_{n}(x)\right) .
\end{gathered}
$$

We next prove that $\omega$ is admissible. With respect to mini-squares of the form

$$
\begin{aligned}
& { }^{(n, 0)} \swarrow \searrow^{(0, m)} \\
& (0, m) \searrow{ }_{(n, 0)}
\end{aligned}
$$

condition (8.5.ii) takes the form $\omega_{T}(m, z)=\omega_{T}\left(m, S^{n}(z)\right)$, while for mini-squares of the form

$$
\begin{aligned}
& (0, m) \swarrow \searrow^{(n, 0)} \\
& (n, 0) \searrow \swarrow^{(0, m)}
\end{aligned}
$$

it becomes $\omega_{S}(n, z)=\omega_{S}\left(n, T^{m}(z)\right)$. That $\omega$ is admissible then follows immediately from Lemma (10.3).

It remains to prove (2.1). So let $(n, m) \in \mathbb{N} \times \mathbb{N}$ and $y \in X$. Then

$$
\begin{gathered}
\sum_{\theta_{(n, m)}(x)=y} \omega((n, m), x)=\sum_{S^{n}\left(T^{m}(x)\right)=y} \omega_{S}(n, x) \omega_{T}(m, x)= \\
=\sum_{S^{n}(w)=y} \sum_{T^{m}(x)=w} \omega_{S}\left(n, T^{m}(x)\right) \omega_{T}(m, x)=\sum_{S^{n}(w)=y} \omega_{S}(n, w) \sum_{T^{m}(x)=w} \omega_{T}(m, x)=1 .
\end{gathered}
$$




\section{Ledrappier's shift.}

We now wish to give an interesting concrete example of a pair of star-commuting endomorphisms of Bernoulli's space. Let us first recall the construction of Ledrappier's dynamical system [9], which is also discussed in [2]. Let

$$
K=\{0,1\}^{\mathbb{N}^{2}}
$$

have the product topology and let $X$ be the subset of $K$ formed by the elements $x \in K$ such that

$$
x_{n, m}+x_{n+1, m}+x_{n, m+1}=0, \quad \forall(n, m) \in \mathbb{N}^{2},
$$

where addition is performed modulo 2. Clearly $X$ is a compact subspace of $K$ which is invariant under both the horizontal and vertical shifts, namely the transformations

$$
H, V: K \rightarrow K
$$

given by

$$
H(x)_{n, m}=x_{n+1, m} \quad \text { and } \quad V(x)_{n, m}=x_{n, m+1} .
$$

Our short term goal is to show that the restrictions of $H$ and $V$ to $X$ give a pair of star-commuting endomorphisms, for which we may then apply the results of section (10).

Let us view the elements of $K$ as possible ways of arranging zeros and ones along the vertices of the lattice $\mathbb{N}^{2}$. It is easy to see that $X$ consists precisely of such arrangements which are entirely made of the following four patterns

$\begin{array}{cccccccc}. & . & . & . & . & . & . & . \\ . & 0 & . & . & . & 1 & . & . \\ . & 0 & 0 & . & . & 0 & 1 & . \\ . & . & . & . & . & . & . & . \\ & & & & & & & \\ . & . & . & . & . & . & . & . \\ . & 1 & . & . & . & 0 & . & . \\ . & 1 & 0 & . & . & 1 & 1 & . \\ . & . & . & . & . & . & . & .\end{array}$

If on $(\dagger)$ we perform the change of variables $(p, q)=(n, m+1)$, we may rewrite it as

$$
x_{p, q}=-x_{p, q-1}-x_{p+1, q-1}, \quad \forall p \in \mathbb{N}, \quad \forall q \geq 1,
$$

which says that if $x \in X$, then each entry of $x$ is determined by the entry immediately below it and the one to the right of that. In particular each row of $x$ is determined by the row below it and hence the first row determines everything. In other words the map

$$
\left\{x_{n, m}\right\}_{n, m \in \mathbb{N}} \in X \longmapsto\left\{x_{n, 0}\right\}_{n \in \mathbb{N}} \in \Omega
$$


is a homeomorphism from $X$ onto Bernoulli's space $\Omega=\{0,1\}^{\mathbb{N}}$. It is easy to see that, under this homeomorphism, the horizontal shift $H$ identifies with the usual Bernoulli shift $S$, given by

$$
S:\left\{\omega_{n}\right\}_{n \in \mathbb{N}} \in \Omega \longmapsto\left\{\omega_{n+1}\right\}_{n \in \mathbb{N}} \in \Omega .
$$

Almost as easily one checks that the vertical shift $V$ becomes the map

$$
T:\left\{\omega_{n}\right\}_{n \in \mathbb{N}} \in \Omega \longmapsto\left\{\omega_{n}+\omega_{n+1}\right\}_{n \in \mathbb{N}} \in \Omega .
$$

Observe that $\Omega$ is an abelian group under coordinatewise addition modulo 2. With this group structure one has that $S$ and $T$ are group homomorphism and $T=i d+S$.

11.1. Lemma. Let $G$ be an abelian group, let $\phi: G \rightarrow G$ be a surjective group homomorphism, and let $\psi=\phi+i d$. Then $(\phi, \psi)$ is star-commuting.

Proof. It is obvious that $\phi$ and $\psi$ commute. Next suppose that $\phi(x)=\psi(y)$. Choose $z_{1} \in G$ such that $\phi\left(z_{1}\right)=y$, and notice that $k:=x-y-z_{1} \in \operatorname{Ker}(\phi)$. Indeed

$$
\phi(k)=\phi(x)-\phi(y)-\phi\left(z_{1}\right)=\phi(x)-\phi(y)-y=\phi(x)-\psi(y)=0 .
$$

Setting $z=z_{1}+k$, one obviously has that $\phi(z)=\phi\left(z_{1}+k\right)=\phi\left(z_{1}\right)=y$, and moreover

$$
\psi(z)=\phi(z)+z=y+z_{1}+x-y-z_{1}=x .
$$

In order to show uniqueness, suppose that $z^{\prime} \in G$ is such that $\phi\left(z^{\prime}\right)=y$ and $\psi\left(z^{\prime}\right)=x$. Then

$$
z^{\prime}=\psi\left(z^{\prime}\right)-\phi\left(z^{\prime}\right)=x-y=\psi(z)-\phi(z)=z .
$$

As an immediate corollary we conclude that $(S, T)$ is star-commuting. It is well known that $S$ is an endomorphism of $\Omega$. It can be shown that $T$ is conjugated to $S$ and hence $T$ is also an endomorphism. We therefore find ourselves precisely in the situation of section (10), as desired.

\section{Example: an action of the multiplicative integers.}

Consider the multiplicative group of strictly positive rational numbers $G=\mathbb{Q}_{+}^{\times}$, and the sub-semigroup $P=\mathbb{N} \backslash\{0\}$. It is clear that $(G, P)$ is a lattice-ordered group with

$$
n \vee m=\operatorname{gcd}(n, m)
$$

(greatest common divisor) and

$$
n \wedge m=\operatorname{lcm}(n, m)
$$

(least common multiple).

Observe that, in the present context, the mini-squares are given by ${ }_{m}^{n} \swarrow^{n} \searrow_{n}^{m}$ where $n$ and $m$ are relatively prime. Consider the action $\theta$ of $P$ on the unit circle $S^{1}$ given by

$$
\theta_{n}(x)=x^{n}, \quad \forall x \in S^{1} .
$$

12.1. Proposition. The action $\theta$ defined above is admissible. 
Proof. Let $(n, m, m, n)$ be a mini-square such as the one above and suppose that $x, y \in S^{1}$ are such that $x^{m}=y^{n}$. We need to find $z \in S^{1}$ such that

$$
z^{n}=x, \text { and } \quad z^{m}=y .
$$

Let $w$ be any $n^{\text {th }}$ root of $x$, so that $w^{n}=x$, and notice that

$$
\left(w^{-m} y\right)^{n}=\left(w^{n}\right)^{-m} y^{n}=x^{-m} y^{n}=1
$$

Thus $\lambda:=w^{-m} y$ is an $n^{t h}$ root of 1 . Since $\operatorname{gcd}(n, m)=1$, the mapping $\rho \mapsto \rho^{m}$ is a bijection on the set of all $n^{\text {th }}$ roots of 1 . Thus we may choose an $n^{\text {th }}$ root $\rho$ of 1 such that $\rho^{m}=\lambda$. Setting $z=\rho w$ we have

$$
z^{n}=\rho^{n} w^{n}=x, \quad \text { and } \quad z^{m}=\rho^{m} w^{m}=\rho^{m} \lambda^{-1} y=y,
$$

thus taking care of existence. If $z_{1}$ and $z_{2}$ are elements of $S^{1}$ such that

$$
z_{1}^{n}=x=z_{2}^{n}, \quad \text { and } \quad z_{1}^{m}=y=z_{2}^{m},
$$

write $z_{j}=e^{2 \pi \theta_{j}}$, for $j=1,2$, and observe that

$$
e^{2 \pi n \theta_{1}}=e^{2 \pi n \theta_{2}} \Longrightarrow p:=n\left(\theta_{2}-\theta_{1}\right) \in \mathbb{Z}
$$

and similarly $q:=m\left(\theta_{2}-\theta_{1}\right) \in \mathbb{Z}$. Thus

$$
\frac{p}{n}=\left(\theta_{2}-\theta_{1}\right)=\frac{q}{m}
$$

so that $n q=m p$. Since $\operatorname{gcd}(n, m)=1$, we have that $n$ divides $p$, so that $\theta_{2}-\theta_{1} \in \mathbb{Z}$ and hence $z_{1}=z_{2}$.

There is a very elementary cocycle we can define in the present context:

12.2. Proposition. Setting $\omega(n, x)=1 / n$, we have that $\omega$ is a normalized admissible cocycle which does not vanish anywhere.

Proof. Left to the reader. 


\section{Example: polymorphisms.}

Let $X$ be a compact space. According to [1] a polymorphism

$$
X \stackrel{T}{\leftarrow} \Sigma \stackrel{S}{\rightarrow} X
$$

consists of a pair of surjective local homeomorphisms $S, T: \Sigma \rightarrow X$, where $\Sigma$ is a compact space. The idea is to think of "ST-1 " as a multivalued map from $X$ to $X$.

One special case of interest [1] is when $\Sigma=X$ and $S T=T S$. In this case we may generalize the usual notion of transformation groupoid by introducing the following:

$$
\mathcal{H}=\left\{(x, k, y) \in X \times \mathbb{Z} \times X: \exists n, m \in \mathbb{N}, k=n-m, S^{n} T^{m}(x)=S^{m} T^{n}(y)\right\} .
$$

Note that the definition of the groupoid of a polymorphism given in [1] is not correct and should be replaced by this one.

13.1. Proposition. $\mathcal{H}$ is a groupoid under the operations

$$
(x, k, y)(y, \ell, z)=(x, k+\ell, z), \quad \text { and } \quad(x, k, y)^{-1}=(y,-k, x) .
$$

Proof. Given $(x, k, y)$ and $(y, \ell, z)$ in $\mathcal{H}$, let $k=n_{1}-m_{1}$, and $\ell=n_{2}-m_{2}$, such that

$$
S^{n_{1}} T^{m_{1}}(x)=S^{m_{1}} T^{n_{1}}(y), \text { and } \quad S^{n_{2}} T^{m_{2}}(y)=S^{m_{2}} T^{n_{2}}(z) .
$$

Then

$$
\begin{aligned}
& S^{n_{1}+n_{2}} T^{m_{1}+m_{2}}(x)=S^{n_{2}} T^{m_{2}} S^{n_{1}} T^{m_{1}}(x)=S^{n_{2}} T^{m_{2}} S^{m_{1}} T^{n_{1}}(y)= \\
& =S^{m_{1}} T^{n_{1}} S^{n_{2}} T^{m_{2}}(y)=S^{m_{1}} T^{n_{1}} S^{m_{2}} T^{n_{2}}(z)=S^{m_{1}+m_{2}} T^{n_{1}+n_{2}}(z),
\end{aligned}
$$

proving that

$$
\mathcal{H} \ni\left(x,\left(n_{1}+n_{2}\right)-\left(m_{1}+m_{2}\right), z\right)=(x, k+\ell, z) .
$$

That $(y,-k, x) \in \mathcal{H}$ is obvious. We leave it for the reader to verify the remaining points.

Alternatively consider the action $\theta$ of $\mathbb{N} \times \mathbb{N}$ on $X$ defined near the beginning of section (10) by

$$
\theta_{(n, m)}=S^{n} T^{m}, \quad \forall(n, m) \in \mathbb{N} \times \mathbb{N},
$$

and let $\mathcal{G}$ be the groupoid defined in terms of $\theta$ as in (3.1).

One should not expect $\mathcal{H}$ and $\mathcal{G}$ to coincide since the former should be thought of as the transformation groupoid associated to the dynamical system generated by the "multivalued" map $S T^{-1}$, while the latter is generated by $S$ and $T$. But $\mathcal{H}$ may be viewed as a subgroupoid of $\mathcal{G}$ in the following sense: 
13.2. Proposition. Let $d: \mathcal{G} \rightarrow \mathbb{Z}$ be the groupoid homomorphism given by

$$
d(x,(n, m), y)=n+m .
$$

Then the map

$$
\phi:(x, k, y) \in \mathcal{H} \longmapsto(x,(k,-k), y) \in \mathcal{G}
$$

defines an isomorphism from $\mathcal{H}$ to the kernel of $d$.

Proof. Let us begin by verifying that $\phi(x, k, y)$ does indeed belong to $\mathcal{G}$, for every $(x, k, y) \in$ $\mathcal{H}$. Write $k=n-m$, in such a way that $S^{n} T^{m}(x)=S^{m} T^{n}(y)$. Since this is equivalent to $\theta_{(n, m)}(x)=\theta_{(m, n)}(y)$, we have that

$$
\mathcal{G} \ni(x,(n, m)-(m, n), y)=(x,(k,-k), y) .
$$

The only non obvious remaining point is perhaps to prove that the kernel of $d$ is contained in the range of $\phi$. In order to prove this let $(x,(n, m), y) \in \operatorname{Ker}(d)$, so that $m=-n$. By definition there exists $(p, q),(r, s) \in \mathbb{N} \times \mathbb{N}$ such that

$$
(n, m)=(n,-n)=(p, q)-(r, s), \quad \text { and } \quad \theta_{(p, q)}(x)=\theta_{(r, s)}(y) .
$$

In other words $n=p-r=s-q$, and $S^{p} T^{q}(x)=S^{r} T^{s}(y)$. For any $(k, l) \in \mathbb{N} \times \mathbb{N}$ notice that

$$
S^{k+p} T^{l+q}(x)=S^{k} T^{l} S^{p} T^{q}(x)=S^{k} T^{l} S^{r} T^{s}(y)=S^{k+r} T^{l+s}(y) .
$$

Choose $l \in \mathbb{N}$ big enough for $l+s-p \geq 0$, and set $k=l+s-p$. Then obviously $k+p=l+s$, while

$$
l+q=l+s-p+r=k+r,
$$

thus proving that

$$
\mathcal{H} \ni(x,(k+p)-(l+q), y)=(x, n, y) \text {. }
$$

Moreover

$$
\phi(x, n, y)=(x,(n,-n), y)=(x,(n, m), y) .
$$

Suppose from now on that $(S, T)$ star-commutes. Then $\theta$ is an admissible action by (10.2), and hence $\mathcal{G}$ is a locally compact étale groupoid by (3.2). It is clear that $\phi(\mathcal{H})$ is an open subgroupoid of $\mathcal{G}$ and hence $\mathcal{H}$ may be turned into a locally compact étale groupoid with the induced topology. Moreover let $\omega$ be a never vanishing normalized coherent cocycle for $\theta$, such as that given by (10.4). One may then consider the interaction group $(C(X), \mathbb{Z} \times \mathbb{Z}, V)$ given by (2.8). Define

$$
\mu: n \in \mathbb{Z} \longrightarrow(n,-n) \in \mathbb{Z} \times \mathbb{Z},
$$

and let

$$
W_{n}=V_{\mu(n)}, \quad \forall n \in \mathbb{Z} .
$$


An explicit formula for $W_{n}$ is easy to write down:

$$
\left.W_{n}(f)\right|_{y}= \begin{cases}\sum_{T^{n}(x)=y} \omega(n, x) f\left(S^{n}(x)\right), & \text { if } n \geq 0, \\ \sum_{S^{-n}(x)=y} \omega(-n, x) f\left(T^{-n}(x)\right), & \text { if } n<0,\end{cases}
$$

for every $f \in C(X)$, and $y \in X$. For example, $\left.W_{1}(f)\right|_{y}$ is the weighted average of $f(z)$ as $z$ runs in the finite set $S\left(T^{-1}(\{y\})\right)$.

$W_{1}(f)$ is therefore the single valued function that best mimics the composition of $f$ with the multivalued function $S T^{-1}$. The latter being precisely what one wants to capture by considering the polymorphism $X \stackrel{T}{\leftarrow} \Sigma \stackrel{S}{\rightarrow} X$.

It is then obvious that $(C(X), \mathbb{Z}, W)$ is an interaction group. It is interesting to notice that, contrary to what happens to $V$, and unless either $T$ or $S$ are invertible maps, there is no $n \in \mathbb{Z}$ for which $W_{n}$ is an endomorphism of $C(X)$.

13.3. Theorem. There is a natural isomorphism

$$
\phi: C(X) \rtimes_{W} \mathbb{Z} \rightarrow C^{*}(\mathcal{H})
$$

Proof. The proof follows essentially the same method used to prove (6.6), so we restrict ourselves here to a brief outline.

First observe that $\mathcal{H}$ is open in $\mathcal{G}$, so $C^{*}(\mathcal{H})$ is naturally a subalgebra of $C^{*}(\mathcal{G})$. Considering the covariant representation $(\pi, \sigma)$ given by $(6.1)$ it is immediate to verify that $(\pi, \sigma \circ \mu)$ is a covariant representation of $(C(X), \mathbb{Z}, W)$, which actually takes values within $C^{*}(\mathcal{H})$. That it is strongly covariant is an immediate consequence of $(\pi, \sigma)$ possessing this property. We therefore obtain a *homomorphism

$$
\pi \times(\sigma \circ \mu): C(X) \rtimes_{W} \mathbb{Z} \rightarrow C^{*}(\mathcal{H})
$$

which is injective by the same reasons used in (6.1). Surjectivity also follows as before. 


\section{A counter-example.}

In this section we want to show an example of a semigroup action which does not admit a never vanishing coherent cocycle.

Let us begin by introducing some notation: given a compact space $X$ and a function $S: X \rightarrow X$ define an equivalence relation $R_{S}$ on $X$ by

$$
(x, y) \in R_{S} \Longleftrightarrow S(x)=S(y) .
$$

Recall that if $R_{1}$ and $R_{2}$ are relations on $X$ one defines the composition of $R_{1}$ and $R_{2}$ to be the relation

$$
R_{1} \circ R_{2}=\left\{(x, z) \in X \times X: \exists y \in X,(x, y) \in R_{1},(y, z) \in R_{2}\right\} .
$$

We will say that $R_{1}$ and $R_{2}$ commute if $R_{1} \circ R_{2}=R_{2} \circ R_{1}$.

14.1. Proposition. Let $G$ be a group, $P$ be a subsemigroup of $G$, and $\theta: P \rightarrow \operatorname{End}(X)$ be a semigroup action. If $\omega$ is a never vanishing coherent cocycle for $\theta$, then $R_{\theta_{n}}$ and $R_{\theta_{m}}$ commute for every $n, m \in P$.

Proof. Observe that $(x, z) \in R_{\theta_{n}} \circ R_{\theta_{m}}$ if and only if $C_{x, z}^{n, m} \neq \emptyset$ (see (2.6) for a definition of $\left.C_{x, z}^{n, m}\right)$. Given $(x, z) \in R_{\theta_{n}} \circ R_{\theta_{m}}$ we then have that $W_{m}\left(C_{x, z}^{n, m}\right)>0$, because $\omega$ is never zero. Since $\omega$ is coherent we deduce from (2.7) that $W_{n}\left(C_{x, z}^{m, n}\right)>0$, and hence that $C_{x, z}^{m, n} \neq \emptyset$, from were we have that $(x, z) \in R_{\theta_{m}} \circ R_{\theta_{n}}$. This shows that

$$
R_{\theta_{n}} \circ R_{\theta_{m}} \subseteq R_{\theta_{m}} \circ R_{\theta_{n}}
$$

The converse inclusion follows similarly.

In order to exhibit a semigroup action which does not admit a never vanishing coherent cocycle it is therefore enough to provide one for which the conclusion of the above result fails.

Given commuting endomorphisms $S, T \in \operatorname{End}(X)$, define a semigroup action of $\mathbb{N} \times \mathbb{N}$ on $X$ by

$$
\theta:(n, m) \in \mathbb{N} \times \mathbb{N} \mapsto S^{n} T^{m} \in \operatorname{End}(X),
$$

as in section (10). Our plan is thus to provide endomorphisms $S$ and $T$ for which $R_{S}$ and $R_{T}$ do not commute.

For every $p \in \mathbb{N}$, let $\Omega_{p}:=\{0,1\}^{p}$ and let $\Omega=\Omega_{\infty}=\{0,1\}^{\mathbb{N}}$. With the product topology $\Omega$ is a compact space also known as Bernoulli's space. Let $S$ be the shift on $\Omega$, namely the transformation

$$
S:\left(x_{0}, x_{1}, x_{2}, \ldots\right) \in \Omega \longmapsto\left(x_{1}, x_{2}, x_{3}, \ldots\right) \in \Omega .
$$

It is well known that $S$ is an endomorphisms of $\Omega$. 
We next wish to describe a class of transformations $T: \Omega \rightarrow \Omega$ which commute with $S$. For this let $p \in \mathbb{N}$ and choose any subset $D \subseteq \Omega_{p}$, henceforth referred to as the dictionary. For $x=\left(x_{0}, x_{1}, x_{2}, \ldots\right) \in \Omega$ define

$$
T(x)_{k}= \begin{cases}1, & \text { if }\left(x_{k}, x_{k+1}, \ldots, x_{k+p-1}\right) \in D \\ 0, & \text { otherwise. }\end{cases}
$$

In other words, to compute $T(x)$ one slides a window of width $p$ along $x$ and checks whether or not the word seen through the window belongs to the dictionary. Recording the answers as a sequence of ones (when the word belongs to $D$ ) and zeros (otherwise), we obtain $T(x)$.

$$
\left(\begin{array}{l}
\text { initial window position } \\
\hdashline x_{0}, x_{1}, x_{2}, \ldots, x_{p-1},
\end{array}, x_{p}, \ldots, x_{k}, x_{k+1}, \ldots, x_{k+p-1}, x_{k+p}, \ldots\right)
$$

The sliding window method.

It is easy to see that $T$ is a continuous mapping which commutes with $S$. Such transformations are sometimes called cellular automata. By a well known result due to Hedlund [8], any continuous map $T: \Omega \rightarrow \Omega$ which commutes with $S$ has the above form for a suitable dictionary $D$.

We now wish to describe a specific class of dictionaries for which the associated cellular automaton is a surjective local homeomorphism.

14.2. Definition. We shall say that a given subset $D \subseteq \Omega_{p}$ is progressive if for every $\beta \in \Omega_{p-1}$ there exists a unique $\varepsilon \in\{0,1\}$ such that $\beta \varepsilon \in D$ (concatenation).

Notice that when $D$ is progressive and we are about to choose the last component of a word which already has $p-1$ components, say

$$
\beta=\left(x_{0}, \ldots, x_{p-2}, ?\right)
$$

we always have the option of forming a word which belongs to $D$ or not.

14.3. Theorem. Let $T$ be a cellular automaton associated to a progressive dictionary $D$. Then $T$ is a surjective local homeomorphism, that is, $T \in \operatorname{End}(\Omega)$.

Proof. For the duration of this proof we shall find it useful to extend $T$ to finite words, obtaining, for every $m \geq p$, a map

$$
T_{m}: \Omega_{m} \rightarrow \Omega_{m-p+1},
$$

defined by the above sliding window method. 
Given $y \in \Omega$, let $\beta=\left(x_{0}, \ldots, x_{p-2}\right)$ be any word in $\Omega_{p-1}$. Since $D$ is progressive there exists a unique $x_{p-1} \in\{0,1\}$ such that

$$
\beta_{0}:=\left(x_{0}, \ldots, x_{p-2}, x_{p-1}\right) \in D \Longleftrightarrow y_{0}=1 \text {. }
$$

One then has that $T_{p}\left(\beta_{0}\right)$ is the single-letter word $\left(y_{0}\right)$. Again because $D$ is progressive there exists a unique $x_{p} \in\{0,1\}$ such that

$$
\left(x_{1}, \ldots, x_{p-1}, x_{p}\right) \in D \Longleftrightarrow y_{1}=1 \text {. }
$$

Thus

$$
\beta_{1}:=\left(x_{0}, x_{1}, \ldots, x_{p-1}, x_{p}\right) \in \Omega_{p+1},
$$

and $T_{p+1}\left(\beta_{1}\right)=\left(y_{0}, y_{1}\right)$. By continuing in this fashion one builds an infinite word $x \in \Omega$ such that $T(x)=y$, hence proving that $T$ is surjective.

Observe that, after the initial choice of $\beta$, each $x_{k}$ (for $k \geq p-1$ ) was uniquely determined. Therefore the restriction of $T$ to

$$
V_{\beta}=\left\{x \in \Omega: x_{i}=\beta_{i}, \forall i=0, \ldots, p-2\right\},
$$

gives a bijection onto $\Omega$. Since $V_{\beta}$ is compact we conclude that $\left.T\right|_{V_{\beta}}$ is a homeomorphism. Moreover the collection $\left\{V_{\beta}\right\}_{\beta \in \Omega_{p-1}}$ is an open cover of $\Omega$, and hence $T$ is a local homeomorphism.

14.4. Example. Consider the progressive set

$$
D=\{000,100,010,111\} \subseteq \Omega^{3},
$$

and let $T$ be the cellular automaton associated to $D$. Then $(S, T)$ is a pair of endomorphisms of $\Omega$ such that $R_{S}$ and $R_{T}$ do not commute.

Proof. Let $x, y, z \in \Omega$ be given by

$$
x=0111111 \ldots, \quad y=111111 \ldots, \text { and } z=0000000
$$

Then

$$
S(x)=S(y)=T(y)=T(z)=11111 \ldots
$$

so $(x, y) \in R_{S}$, and $(y, z) \in R_{T}$, whence $(x, z) \in R_{S} \circ R_{T}$. We claim that $(x, z) \notin R_{T} \circ R_{S}$. By contradiction suppose otherwise so there exists $y^{\prime}$ such that

$$
T(x)=T\left(y^{\prime}\right), \text { and } \quad S\left(y^{\prime}\right)=S(z) .
$$

The second equation allows for only two choices for $y^{\prime}$, namely

$$
y_{1}^{\prime}=0000000 \ldots, \text { and } y_{2}^{\prime}=1000000 \ldots
$$

none of which satisfy $T(x)=T\left(y^{\prime}\right)$.

14.5. Corollary. Let $S$ and $T$ be as in (14.4) and define a semigroup action of $\mathbb{N} \times \mathbb{N}$ on $\Omega$ by

$$
\theta:(n, m) \in \mathbb{N} \times \mathbb{N} \mapsto S^{n} T^{m} \in \operatorname{End}(\Omega) .
$$

Then there does not exist a never vanishing coherent cocycle for $\theta$.

It seems that this example is as odds with the last Proposition in [2]. 


\section{REFERENCES}

[1] V. Arzumanian and J. Renault, "Examples of pseudogroups and their C*-algebras", in Operator algebras and quantum field theory (Rome, 1996), 93-104, Internat. Press, Cambridge, MA, 1997.

[2] V. Deaconu, "Groupoids associated with endomorphisms", Trans. Amer. Math. Soc., 347 (1995), 1779-1786.

[3] V. Deaconu, "C*-algebras of commuting endomorphisms", preprint, arXiv:math.OA/0406624.

[4] R. Exel, "Amenability for Fell bundles", J. reine angew. Math., 492 (1997), 41-73, arXiv:funct an/9604009.

[5] R. Exel, "A new look at the crossed-product of a C*-algebra by an endomorphism", Ergodic Theory Dynam. Systems, 23 (2003), 1733-1750, arXiv:math.OA/0012084].

[6] R. Exel, "A new look at the crossed-product of a $\mathrm{C}^{*}$-algebra by a semigroup of endomorphisms", preprint, Universidade Federal de Santa Catarina, 2005, arXiv:math.OA/0511061.

[7] R. Exel and A. Vershik, "C*-algebras of irreversible dynamical systems", Canadian Mathematical Journal, 58 (2006), 39-63, arXiv:math.OA/0203185.

[8] G. A. Hedlund, "Endormorphisms and automorphisms of the shift dynamical system", Math. Systems Theory, 3 (1969), 320-375.

[9] F. Ledrappier, "Un champ markovien peut être d'entropie nulle et mélangeant", C. R. Acad. Sci. Paris Sér. A-B, 287 (1978), no. 7, A561-A563.

[10] J. Renault, "A groupoid approach to $C^{*}$-algebras", Lecture Notes in Mathematics vol. 793, Springer, 1980.

[11] T. Yeend, "Groupoid models for the $\mathrm{C}^{*}$-algebras of topological higher-rank graphs", J. Oper. Theory, to appear.

Departamento de MATEmática

Universidade Federal de Santa Catarina

88040-900 - FLORIANÓPOLIS - BRASIL

exel@mtm.ufsc.br
DÉPARTMEnT DE MAThÉmatiQUes

UNIVERSITÉ D'ORLÉANS

45067 OrLÉANs, FrANCE

Jean.Renault@univ-orleans.fr 